\title{
Euclidean extensions of dynamical systems *
}

\author{
Matthew Nicol \\ Department of Mathematics and Statistics \\ University of Surrey \\ Guildford GU2 7XH, UK
}

\author{
Ian Melbourne \\ Department of Mathematics \\ University of Houston \\ Houston, TX 77204-3476, USA
}

\author{
Peter Ashwin \\ School of Mathematical Science \\ University of Exeter \\ Exeter EX4 4QJ, UK
}

November 2, 2000

\begin{abstract}
We consider special Euclidean $(\mathbf{S E}(n))$ group extensions of dynamical systems and obtain results on the unboundedness and growth rates of trajectories for smooth extensions. The results depend on $n$ and the base dynamics considered.

For discrete dynamics on the base with a dense set of periodic points, we prove unboundedness of trajectories for generic extensions provided $n=2$ or $n$ is odd. If in addition the base dynamics is Anosov, then generically trajectories are unbounded for all $n$, exhibit square root growth, and converge in distribution to a nondegenerate standard $n$-dimensional normal distribution.

For sufficiently smooth $\mathbf{S E}(2)$-extensions of quasiperiodic flows, we prove that trajectories of the group extension are typically bounded in a probabilistic sense, but there is a dense set of base rotations for which extensions are typically unbounded in a topological sense. The results on unboundedness are generalised to $\mathbf{S E}(n)$ ( $n$ odd) and to extensions of quasiperiodic maps.

We obtain these results by exploiting the fact that $\mathbf{S E}(n)$ has the semi-direct product structure $\Gamma=G \ltimes \mathbb{R}^{n}$ where $G$ is a compact connected Lie group and $\mathbb{R}^{n}$ is a normal abelian subgroup of $\Gamma$. This means that our results also apply to extensions by this wider class of groups.
\end{abstract}

*To appear in Nonlinearity 


\section{Contents}

1 Introduction 2

2 Maps and flows $\quad 6$

(a) The group $\Gamma=G \ltimes \mathbb{R}^{n} \ldots \ldots \ldots \ldots$

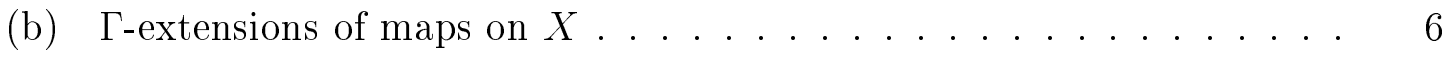

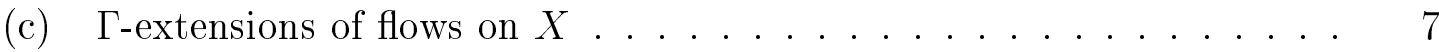

3 Unboundedness of trajectories: Linear and sublinear drift 8

(a) Some results on linear drift . . . . . . . . . . . 10

4 Extensions of chaotic base dynamics 12

(a) Topological dynamics . . . . . . . . . . . . . . . . . . . . 12

(b) Unboundedness of deviations . . . . . . . . . . . . . . . 14

5 Equivariant deterministic central limit theorems $\quad 15$

(a) An $n$-dimensional equivariant central limit theorem . . . . . . . . 16

(b) An equivariant Livšic Theorem . . . . . . . . . . . . . 19

(c) Nondegenerate central limit theorems . . . . . . . . . . . . . . . . 23

(d) Special cases: $G=\mathbf{1}$ and $G=\mathbf{S O}(n) \ldots \ldots . \ldots 24$

6 Extensions of hyperbolic base dynamics 25

7 Extensions of quasiperiodic base dynamics 26

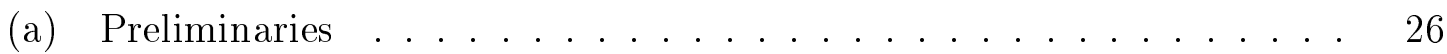

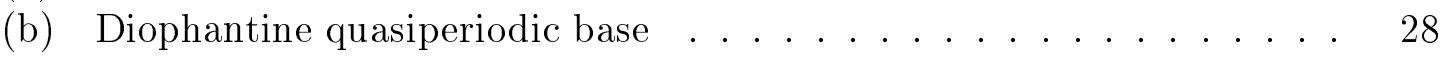

(c) Non-Diophantine quasiperiodic base . . . . . . . . . . . . 30

\section{Introduction}

Recently there has been substantial progress in understanding the ergodic and mixing properties of generic compact group extensions of dynamical systems. In contrast very little is known about extensions by noncompact groups; nevertheless noncompact groups are of great interest in applications. The setting raises natural questions about the unboundedness and growth rates of typical orbits in generic extensions.

In this paper we investigate the issues of unboundedness and growth rates for a certain class of noncompact group extensions of dynamical systems. The class of groups includes the special Euclidean group $\Gamma=\mathbf{S E}(n)=\mathbf{S O}(n) \ltimes \mathbb{R}^{n}$ consisting of 
rotations $\mathbf{S O}(n)$ and translations $\mathbb{R}^{n}$ in $n$-dimensional space. Throughout the introduction, we emphasize the special Euclidean group $\Gamma=\mathbf{S E}(n), n \geq 2$, contrasting the results with the comparatively well understood case $\Gamma=\mathbb{R}^{n}, n \geq 1$. However, the methods in this paper easily generalise to the case of noncompact connected Lie groups of the form $\Gamma=G \ltimes \mathbb{R}^{n}$ (semidirect product) where $G$ is a compact connected subgroup of $\Gamma$ and $\mathbb{R}^{n}$ is an abelian normal subgroup of $\Gamma$.

Recall that a group extension ( $\Gamma$-extension) of a base dynamical system consisting of a smooth mapping or flow on a compact manifold $X$ is defined on the product space $X \times \Gamma$. Such extensions arise naturally in dynamical systems that are equivariant with respect to a symmetry group $\Gamma$ and have been extensively studied both by ergodic theorists and in the equivariant dynamics literature. We take the approach (common in this literature) that we specify the dynamics on $X$ and then investigate the generic behavior of the extension.

Given a semidirect product structure $\Gamma=G \ltimes \mathbb{R}^{n}$, any $\Gamma$-extension is an $\mathbb{R}^{n}$ extension of a $G$-extension. (That is, any $\Gamma$-extension has a $G$-extension as a factor.) We will use this as a tool to extend compact group extension results to the noncompact case. In the case $\Gamma=\mathbb{R}^{n}$, the $\Gamma$-extension reduces to the well-studied case $X \times \mathbb{R}^{n}$. In the case $\Gamma=\mathbf{S E}(n)$, we have a $\Gamma$-extension on $(X \times \mathbf{S O}(n)) \times \mathbb{R}^{n}$. On a cautionary note, it must be emphasized that the $\mathbb{R}^{n}$-extension of $X \times \mathbf{S O}(n)$ is not arbitrary, but is $\mathbf{S O}(n)$-equivariant. That is, $\mathbb{R}^{n}$-extensions $k: X \times \mathbf{S O}(n) \rightarrow \mathbb{R}^{n}$ are required to satisfy

$$
k(x, g)=g k(x, e), \quad \text { for all } g \in \mathbf{S O}(n),
$$

where $e$ denotes the identity element of $\mathbf{S O}(n)$ and $g k(\cdot)$ denotes matrix multiplication of the $n \times n$ matrix $g$ and the $n$-dimensional vector $k(\cdot)$. This restriction reflects the semidirect product structure of $\mathbf{S E}(n)$.

Our main concern in this paper is the unboundedness of trajectories on $X \times \Gamma$, which reduces to unboundedness of their $\mathbb{R}^{n}$ component since $G$ is compact. Our results depend in a fundamental way on properties of the base dynamics and on the group $\Gamma$.

We consider two types of base dynamics; "chaotic" dynamics and quasiperiodic dynamics. (The case of periodic base dynamics is discussed in Ashwin and Melbourne [1] and Wulff et al. [16, Section 4(b)].)

"Chaotic" base dynamics (Section 4) Suppose that the dynamics on $X$ is chaotic in the sense that periodic points are dense in $X$ (we exclude the case that $X$ is itself a periodic orbit). Regardless of ergodic properties of the transformation on $X$, we prove that generic extensions have trajectories that are unbounded when $\Gamma=\mathbb{R}^{n}$ (for all $n$ ) and when $\Gamma=\operatorname{SE}(n)$ (for $n=2$ or $n$ odd, cf. Remark 1.1). 
Suppose further that $\mu$ is an ergodic measure on $X$ and that periodic points are dense in the support of $\mu$. When $\Gamma=\mathbb{R}^{n}$, typically trajectories exhibit linear growth on average, and moreover the deviation from linear growth is unbounded.

If we replace $\Gamma=\mathbb{R}^{n}$ by $\Gamma=\mathbf{S E}(n)$, it is necessary to assume that ergodicity on $X$ lifts to the compact group extension on $X \times \mathbf{S O}(n)$. The validity of this assumption is the subject of extensive current research (see the references in subsection 3(a)) and it is known, for example, that a generic set of extensions are ergodic when $\mu$ is supported on an Axiom A attractor. Under the assumption that ergodicity lifts to $X \times \mathbf{S O}(n)$, we prove for all $n \geq 2$ that trajectories exhibit sublinear growth. (Of course, when $n=1, \mathbf{S E}(1)=\mathbb{R}$ and we expect linear growth.) Combined with our results on unboundedness, we can prove unbounded sublinear growth for $n=2$ or $n$ odd.

Uniformly hyperbolic base dynamics (Section 6) In the case that the dynamics on $X$ is Axiom A, it is known [14] that sums of real observables typically obey central limit theorems. This can be rephrased as saying that for an $\mathbb{R}$-extension, typically the deviation of trajectories from linear growth exhibits square root growth, and there is convergence to a nondegenerate normal distribution. Similarly, for an $\mathbb{R}^{n}$-extension, there is convergence to a general $n$-dimensional normal distribution.

The results for $\mathbf{S E}(n)$-extensions are considerably more difficult and we require recent results of Field and Parry [6], Burns and Wilkinson [4], Dolgopyat [5], and Liverani [10]. We prove that for $n \geq 3$, typically there is convergence to a nondegenerate standard $n$-dimensional normal distribution. The same result holds for $n=2$ provided that $X$ is Anosov on an infranilmanifold.

In proving this result, we strengthen and generalize results of Biktashev and Holden [2] relating chaotic base dynamics to random walks in the translation variables for $\mathbf{S E}(2)$-equivariant problems. In particular we address the question of the degeneracy or nondegeneracy of the normal distribution.

Quasiperiodic base dynamics (Section 7) Suppose that $X=T^{m}$ is a torus and that the dynamics on $X$ is a linear flow $\dot{\theta}=\alpha$ or translation $\theta \mapsto \theta+\alpha$, where $\alpha \in \mathbb{R}^{m}$ is such that all orbits are dense in $X$. If $\Gamma=\mathbb{R}^{n}(n \geq 1)$ it is well-known (and easy to prove) that there is an open and dense full measure set of extensions whose trajectories exhibit linear growth. The growth arises from the constant term (which is typically nonzero) in the Fourier expansion of the $\mathbb{R}^{n}$-extension. If $\alpha$ is Diophantine (and the $\mathbb{R}^{n}$-extension is smooth enough) then the deviation from linear growth is bounded.

The situation for $\Gamma=\operatorname{SE}(n)$ is less straightforward, since it is no longer typically the case that the constant term of the Fourier expansion of the $\mathbb{R}^{n}$-extension is nonzero 
(due to the constraint (1.1)). First, consider SE(2)-extensions of a quasiperiodic flow. If $\alpha$ is Diophantine (which occurs with probability one) we prove that trajectories are bounded for almost all sufficiently smooth extensions. In the non-Diophantine case, we prove that trajectories are generically unbounded in the $C^{r}$ topology for all $r \geq 1$.

Our results on unboundedness in the non-Diophantine case hold also for $\operatorname{SE}(n)$ extensions of flows and maps provided $n$ is odd. However, our results in the Diophantine case are presently restricted to $\mathbf{S E}(2)$-extensions of flows.

Remark 1.1 Many of the results that we have discussed for $\mathbf{S E}(n)$-extensions are restricted to the cases $n=2$ or $n$ odd. The reason for this is that our arguments rely on the existence of a $C^{r}$ small perturbation of the $\mathbf{S E}(n)$-extension of a periodic point in $X$ such that the dynamics of the extension is unbounded. This is easy when $n$ is odd, since extensions of periodic orbits are typically unbounded [1]. When $n=2$, we exploit the fact that $\mathbf{S O}(2)$ is abelian to show that a small perturbation suffices if the period of the periodic orbit is high enough.

We conjecture that similar results hold for all $n$. However, the difficulties that arise for $n$ even, $n \geq 4$, remain unresolved when $X$ is not Axiom A.

Equivariant deterministic central limit theorems (Section 5) In proving our results on $\mathbf{S E}(n)$-extensions of uniformly hyperbolic base dynamics (as described in Section 6), the key step is proving an equivariant deterministic central limit theorem. This is a result about the statistics of compact group extensions $X \times G$ (where $G$ is an arbitrary compact connected Lie group), and is of interest in its own right. Here, we have made extensive use of recent results of Dolgopyat [5] and Liverani [10] which together imply in many cases the validity of deterministic central limit theorems on the partially hyperbolic set $X \times G$. Our main contribution in this area is to show that for equivariant observations (cf. equation (1.1)), nondegeneracy in the central limit theorem is equivalent to unboundedness of trajectories. We show this by means of an equivariant version of the Livšic Theorem [11].

The remainder of this paper is organized as follows. In Section 2, we describe the implications that the semi-direct product structure of $\Gamma=\mathbf{S E}(n)$ has for the structure of the $\mathbf{S E}(n)$-extensions. Indeed similar observations hold for all extensions by groups which are of form $\Gamma=G \ltimes \mathbb{R}^{n}$. In Section 3, we raise questions about the unboundedness of trajectories of $\Gamma$-extensions, distinguishing between linear and sublinear growth of unbounded trajectories, and we summarize results on whether the growth is linear or sublinear.

Our main results can be found in Sections 4-7. In Sections 4, 6 and 7, we consider extensions when the base is respectively chaotic, uniformly hyperbolic and quasiperiodic. Our results on equivariant central limit theorems are given in Section 5 . 


\section{Maps and flows}

In this section, we discuss the general setting for our results. We assume a semidirect product structure of the group $\Gamma$ and discuss the structure of $\Gamma$-extensions of base dynamics on $X$ for dynamics generated by maps and flows.

\section{(a) The group $\Gamma=G \ltimes \mathbb{R}^{n}$}

Throughout this paper, we consider Lie groups $\Gamma$ that are a semidirect product of a connected compact subgroup $G$ and an abelian normal subgroup $\mathbb{R}^{n}$. We write ${ }^{1}$ $\Gamma=G \ltimes \mathbb{R}^{n}$ and note that

$$
\left(g_{1}, v_{1}\right) \cdot\left(g_{2}, v_{2}\right)=\left(g_{1} g_{2}, v_{1}+\rho_{g_{1}} v_{2}\right),
$$

where $g_{1}, g_{2} \in G, v_{1}, v_{2} \in \mathbb{R}^{n}$ and $\rho: G \rightarrow \operatorname{Aut}\left(\mathbb{R}^{n}\right)$. Of course, if $\Gamma$ is the special Euclidean group $\mathbf{S E}(n)$ then we have $G=\mathbf{S O}(n)$ and $\rho_{g} v=g v$ (multiplication of the orthogonal matrix $g$ and the vector $v$ ).

\section{(b) $\Gamma$-extensions of maps on $X$}

Let $f: X \rightarrow X$ be a $C^{k}$ map of a compact manifold $(k \geq 0)$. A $\Gamma$-extension of $f$ is a map $T: X \times \Gamma \rightarrow X \times \Gamma$ defined by

$$
T(x, \gamma)=(f(x), \gamma \zeta(x))
$$

where $\zeta \in C^{r}(X, \Gamma)(r \geq 0)$. Note that the skewing function $\zeta$ acts on the right, and hence the extension $T$ is $\Gamma$-equivariant with respect to the left action of $\Gamma$ where $\delta \cdot(x, \gamma)=(x, \delta \gamma)$ for $\delta \in \Gamma$. Indeed, every $\Gamma$-equivariant mapping $T: X \times \Gamma \rightarrow X \times \Gamma$ has the form $T(x, \gamma)=(f(x), \gamma \zeta(x))$ where $f: X \rightarrow X$ and $\zeta: X \rightarrow \Gamma$.

Given the structure $\Gamma=G \ltimes \mathbb{R}^{n}$, we can write

$$
T(x, g, v)=\left(f(x), g h(x), v+\rho_{g} k(x)\right),
$$

where $h \in C^{r}(X, G)$ and $k \in C^{r}\left(X, \mathbb{R}^{n}\right)$. If we write $S$ as the $G$-extension

$$
S(x, g)=(f(x), g h(x))
$$

then $T$ can be viewed as an $\mathbb{R}^{n}$-extension of the $G$-extension $S$.

On iterating a $\Gamma$-extension of a map, the extension determines cocycles $h_{j}(x)$ and $k_{j}(x)$ characterized by the following Proposition.

\footnotetext{
${ }^{1}$ As is traditional, we use multiplicative notation for the group $G$ and additive for the abelian group $\mathbb{R}^{n}$.
} 
Proposition 2.1 For $j \in \mathbb{N}$ we can write

$$
T^{j}(x, g, v)=\left(f^{j}(x), g h_{j}(x), v+\rho_{g} k_{j}(x)\right),
$$

where the cocycles $h_{j}$ and $k_{j}$ are defined by

$$
\begin{aligned}
& h_{j}(x)=h(x) h(f(x)) \cdots h\left(f^{j-1}(x)\right), \\
& k_{j}(x)=k(x)+\rho_{h_{1}(x)} k(f(x))+\cdots+\rho_{h_{j-1}(x)} k\left(f^{j-1}(x)\right) .
\end{aligned}
$$

Similarly, if $f$ is invertible we can define $h_{j}$ and $k_{j}$ for $j \in \mathbb{Z}$.

Proof This is an elementary induction argument.

\section{(c) $\Gamma$-extensions of flows on $X$}

Suppose that $X$ is a smooth compact manifold and that $f: X \rightarrow T X$ is a $C^{k}$ vector field, $k \geq 1$. Let $L \Gamma$ denote the Lie algebra of $\Gamma$. We consider $\Gamma$-extensions of the form

$$
\dot{x}=f(x), \quad \dot{\gamma}=\gamma \zeta(x),
$$

where $\zeta \in C^{r}(X, L \Gamma)$.

Just as in the case of maps, we exploit the structure $\Gamma=G \ltimes \mathbb{R}^{n}$ by writing equation (2.1) in the form

$$
\dot{x}=f(x), \quad \dot{g}=g h(x), \quad \dot{v}=\rho_{g} k(x),
$$

where $h \in C^{r}(X, L G)$ and $k \in C^{r}\left(X, \mathbb{R}^{n}\right)$. Again the flow of equation (2.2) can be viewed as an $\mathbb{R}^{n}$-extension of a $G$-extension of the flow on $X$.

Remark 2.2 We consider right actions of $\Gamma$ in our definition of $\Gamma$-extensions of maps and flows. These extensions are equivariant under the left action of $\delta \in \Gamma$ on $X \times \Gamma$ by $\delta(x, \gamma)=(x, \delta \gamma)$. In many applications, $X$ is thought of as the orbit space of a group action of $\Gamma$ on, say, a manifold $Y$ and the point $(x, \gamma)$ 'represents' the point $\gamma x$ in the ambient space $Y$. However it is traditional in the ergodic theory literature (but see [6]) to consider group extensions defined by a left action (which would be for maps, $T(x, \gamma)=(f(x), \zeta(x) \gamma))$. As we now explain, these two viewpoints are equivalent for the questions we consider in this paper.

Suppose that $G$ is a compact group with Haar measure $\nu_{G}$ and suppose that $\mu$ is an ergodic measure on $X$. Let $h \in C^{0}(X, G)$. The group extension $T(x, g)=$ $(f(x), g h(x))$ is ergodic with respect to $\mu \times \nu_{G}$ if and only if $T(x, g)=(f(x), h(x) g)$ is 
ergodic (see [6, Theorem 2.4.1] and [12, Theorem 1]). Similar results hold for mixing properties of the extensions.

More generally, if $\zeta$ defines an extension on the right by $T(x, \gamma)=(f(x), \gamma \zeta(x))$ then $\zeta^{-1}$ defines an extension on the left $T^{\prime}(x, \gamma)=\left(f(x), \zeta^{-1}(x) \gamma\right)$ that is topologically conjugate to $T$ under the automorphism $(x, \gamma) \mapsto\left(x, \gamma^{-1}\right)$. Therefore unboundedness and other such properties are independent of which action we choose. Analogous statements hold for group extensions of flows.

\section{Unboundedness of trajectories: Linear and sub- linear drift}

In this section, we raise the question of unboundedness for trajectories on $X \times \Gamma$. By choosing an ergodic measure on the compact group extension $X \times G$, we distinguish between linear growth and sublinear growth. We focus on growth of trajectories for mappings, but the discussion is equally applicable to flows with minor modifications.

Consider the $\Gamma$-extension $T(x, g, v)=\left(f(x), g h(x), v+\rho_{g} k(x)\right)$. The trajectory with initial condition $\left(x_{0}, g_{0}, v_{0}\right)$ is unbounded if the closure of the trajectory is not compact. This is equivalent to unboundedness of the $\mathbb{R}^{n}$ component of the trajectory, since $X$ and $G$ are assumed to be compact. The trajectory with initial condition $\left(x_{0}, g_{0}, v_{0}\right)$ is a translate of the trajectory with initial condition $\left(x_{0}, g_{0}, 0\right)$ so that unboundedness is independent of $v_{0}$. We take $v_{0}=0$ from here on. Let $\left\{\left(x_{N}, g_{N}, v_{N}\right), N \geq 0\right\}$ denote the trajectory with initial condition $\left(x_{0}, g_{0}, 0\right)$ and note that

$$
v_{N}=v_{x_{0}, g_{0}}(N)=\sum_{j=0}^{N-1} \rho_{g_{j}} k\left(x_{j}\right) .
$$

Suppose that $m$ is an ergodic Borel measure on $X \times G$. The Birkhoff ergodic theorem implies that the time averaged linear growth rate

$$
\bar{v}=\lim _{N \rightarrow \infty} \frac{1}{N} v(N)=\lim _{N \rightarrow \infty} \frac{1}{N} \sum_{j=0}^{N-1} \rho_{g_{j}} k\left(x_{j}\right)
$$

exists and is constant for $m$-almost all initial conditions $\left(x_{0}, g_{0}\right) \in X \times G$. We define the deviation from linear growth $\epsilon_{x_{0}, v_{0}}$ by

$$
v_{x_{0}, g_{0}}(N)=\bar{v} N+\epsilon_{x_{0}, g_{0}}(N)
$$

and so for $m$-almost all initial $\left(x_{0}, g_{0}\right)$ we have $\epsilon_{x_{0}, g_{0}}(N)=o(N)$.

The question of the growth of $v_{x_{0}, g_{0}}(N)$ for a given ergodic measure $m$ divides naturally into two subquestions: 
(Q1) Is $\bar{v}$ zero or nonzero?

(Q2) Is $\epsilon(N)$ bounded or unbounded?

Clearly, almost all trajectories are bounded if and only if $\bar{v}=0$ and $\epsilon$ is bounded. Depending on the answers to these questions, we have four mutually exclusive scenarios: (i) bounded growth, (ii) unbounded but sublinear growth, (iii) linear growth with bounded deviation, (iv) linear growth with unbounded deviation.

In the case of periodic base dynamics, there are simple answers to questions (Q1) and (Q2) as shown below in Proposition 3.1. The answer to (Q1) follows from Ashwin and Melbourne [1]. The answer to (Q2) is that the deviation $\epsilon(N)$ from the linear growth is bounded. We note that there is no need to refer to an ergodic measure on $X \times G$ when the dynamics on $X$ is periodic.

Since this result is formulated somewhat differently in [1], and is crucial in later sections of this paper, we rederive the result here. Let $\mathbf{T}$ denote a maximal torus in $G$ and let

$$
\operatorname{Fix}(\mathbf{T})=\left\{v \in \mathbb{R}^{n}: \rho_{g} v=v \text { for all } g \in \mathbf{T}\right\},
$$

denote the fixed-point subspace for that action of $\mathbf{T} \subset G$ on $\mathbb{R}^{n}$. Since all maximal tori are conjugate in $G$, the dimension of $\operatorname{Fix}(\mathbf{T})$ is independent of the choice of $\mathbf{T}$.

Proposition 3.1 Suppose that $P \subset X$ is a periodic solution (or equilibrium) for a flow or map. Let $\mathbf{T}$ be a maximal torus in $G$. Then there is an open and dense set of $\Gamma$-extensions in the $C^{r}$ topology (for each $r \geq 0$ ) such that

(a) If $\operatorname{Fix}(\mathbf{T})=\{0\}$, then all trajectories on $P \times \Gamma$ are bounded.

(b) If $\operatorname{Fix}(\mathbf{T}) \neq\{0\}$, then all trajectories on $P \times \Gamma$ have linear growth with bounded deviation.

Proof We verify the Proposition in the case of maps. Since a periodic point is a fixed point for an iterate of the map $f$, it is sufficient to consider a fixed point $x_{0}$ for $f$. By Proposition 2.1,

$$
v_{x_{0}, g_{0}}(N)=\rho_{g_{0}}\left(I+A+\cdots+A^{N-1}\right) k\left(x_{0}\right),
$$

where $A=\rho_{h\left(x_{0}\right)}$. Write $k\left(x_{0}\right)=y_{0}+y_{1} \in \operatorname{Fix}(A) \oplus \operatorname{Fix}(A)^{\perp}$ and let $B=\left.A\right|_{\operatorname{Fix}(A)^{\perp}}$. Since $A$ is orthogonal and $I-B$ is nonsingular we have

$$
\begin{aligned}
v(N) & =N \rho_{g_{0}} y_{0}+\rho_{g_{0}}\left(I+B+\cdots+B^{N-1}\right) y_{1} \\
& =N \rho_{g_{0}} y_{0}+\rho_{g}(I-B)^{-1}\left(I-B^{N}\right) y_{1} .
\end{aligned}
$$


It follows that $\bar{v}=\rho_{g_{0}} y_{0}$ and that $\epsilon(N)=\rho_{g_{0}}(I-B)^{-1}\left(I-B^{N}\right) y_{1}$ is bounded (since $\|B\|=1$ ). Moreover, $\bar{v}$ is nonzero, generically, if and only if $\operatorname{Fix}(A) \neq\{0\}$. But $A=\rho_{h\left(x_{0}\right)}$ is a general element of $G$ and hence $\operatorname{Fix}(A)$ coincides with $\operatorname{Fix}(\mathbf{T})$ where $\mathbf{T}$ is a maximal torus.

Remark 3.2 For groups of the form $\Gamma=G \ltimes \mathbb{R}^{n}$, and periodic solutions with trivial isotropy, generically $\operatorname{Fix}(\mathbf{T})=\{0\}$ implies compact drift and $\operatorname{Fix}(\mathbf{T}) \neq\{0\}$ implies unbounded drift (using the terminology of [1]). When $\Gamma=\mathbf{S E}(n)$, we obtain compact drift generically for $n$ even and unbounded drift generically for $n$ odd.

\section{(a) Some results on linear drift}

In general, the solutions to questions (Q1) and (Q2) depend on the dynamics on $X$ and the group $\Gamma=G \ltimes \mathbb{R}^{n}$ (specifically, the action $\rho$ of $G$ on $\mathbb{R}^{n}$ ). For certain groups $G$, the solution to (Q1) is elementary and independent of the dynamics. Let $\operatorname{Fix}(G) \subset \mathbb{R}^{n}$ denote the fixed-point subspace for the action of $G$ on $\mathbb{R}^{n}$.

Proposition 3.3 Suppose that $\operatorname{Fix}(G) \neq 0$ and let $m$ be any ergodic measure on $X \times G$. Then there is an open and dense set of $\Gamma$-extensions (for each $r \geq 0$ ) such that $\bar{v} \neq 0$ (linear growth).

Proof By the ergodic theorem, we compute that

$$
\bar{v}=\lim _{N \rightarrow \infty} \frac{1}{N} \sum_{j=0}^{N-1} \rho_{g_{j}} k\left(x_{j}\right)=\int_{X \times G} \rho_{g} k(x) d m(x, g) .
$$

Write $\mathbb{R}^{n}=\operatorname{Fix}(G) \oplus Y_{1}$ where $Y_{1}$ is $G$-invariant. Then $k=k_{0}+k_{1}$, where $k_{0}: X \rightarrow$ $\operatorname{Fix}(G), k_{1}: X \rightarrow Y_{1}$, and

$$
\bar{v}=\int_{X \times G} k_{0}(x) d m(x, g)+\int_{X \times G} \rho_{g} k_{1}(x) d m(x, g) .
$$

We can control the value of the first term by making arbitrarily small constant perturbations to $k_{0}$. Hence, generically $\bar{v} \neq 0$.

It follows from Proposition 3.3 that we expect linear growth for $\Gamma=\mathbb{R}^{n}$, but there is no conclusion for $\Gamma=\mathrm{SE}(n)$. We obtain sharper results by making assumptions on the ergodic measure $m$ on $X \times G$. Let $\nu_{G}$ denote Haar measure on $G$.

Proposition 3.4 Suppose that the invariant measure $m=\mu \times \nu_{G}$ is an ergodic measure on $X \times G$. 
(a) If $\operatorname{Fix}(G)=\{0\}$ then $\bar{v}=0$ (sublinear growth).

(b) If $\operatorname{Fix}(G) \neq\{0\}$ then $\bar{v} \neq 0$ (linear growth) for an open and dense set of $\Gamma$ extensions in the $C^{r}$ topology (for each $r \geq 0$ ).

Proof As in the proof of Proposition 3.3, we compute that

$$
\bar{v}=\lim _{N \rightarrow \infty} \frac{1}{N} \sum_{j=0}^{N-1} \rho_{g_{j}} k\left(x_{j}\right)=\int_{X \times G} \rho_{g} k(x) d \mu(x) d \nu_{G}(g)=\int_{G} \rho_{g} d \nu_{G} \int_{X} k(x) d \mu .
$$

But $\int_{G} \rho_{g} d \nu_{G}$ is orthogonal projection onto $\operatorname{Fix}(G)$. It follows that $\bar{v} \in \operatorname{Fix}(G)$. Moreover $\int_{X} k(x) d \mu$ is a general element of $\mathbb{R}^{n}$ since there are no restrictions on $k$, and hence $\bar{v}$ is a general element of $\operatorname{Fix}(G)$.

By Proposition 3.4, the condition $\operatorname{Fix}(G) \neq\{0\}$ in Proposition 3.3 is sharp provided $\mu \times \nu_{G}$ is an ergodic measure for the compact group extension $X \times G$. (In other words, provided ergodicity lifts to $X \times G$.)

There has been much recent work on the question of the ergodicity of compact group extensions of ergodic dynamical systems. For example, when $G$ is abelian, it follows from Jones and Parry [8] that ergodicity lifts generically in the $C^{0}$ topology. That is, the set of $h \in C^{0}(X, G)$ such that $\mu \times \nu_{G}$ is ergodic on $X \times G$ is residual in the $C^{0}$ topology.

Stronger results are available if the base dynamics is uniformly hyperbolic. Field and Parry [6, Theorem 5.1.1] show that if $G$ is a compact connected Lie group and $\mu$ is an invariant ergodic equilibrium measure on a connected hyperbolic attractor in $X$ then ergodicity lifts generically in the $C^{r}$ topology for all $r \geq 1$. Indeed, there is an open and dense set of $h \in C^{r}(X, G)$ for which the extension is ergodic. (This phenomenon is known as stable ergodicity [7].)

The work of $[6,8]$, combined with Proposition 3.4, yields the following result.

Proposition 3.5 Suppose that $\operatorname{Fix}(G)=\{0\}$ and $\mu$ is an ergodic measure on $X$.

(i) If $G$ is abelian then $m=\mu \times \nu_{G}$ is ergodic and $\bar{v}=0$ for a generic set of extensions in the $C^{0}$ topology.

(ii) If $\mu$ is an invariant ergodic equilibrium measure on a connected hyperbolic attractor in $X$ then $m=\mu \times \nu_{G}$ is ergodic and $\bar{v}=0$ for an open and dense set of extensions in the $C^{r}$ topology (for each $r \geq 1$ ).

Note that when $\Gamma=\operatorname{SE}(n), n \geq 2$, we have $\operatorname{Fix}(G)=\{0\}$ (since $G=\mathbf{S O}(n)$ acts on $\mathbb{R}^{n}$ by matrix multiplication). Hence, the conclusions of Proposition 3.5 apply. Of course, for the group $\Gamma=\operatorname{SE}(1)=\mathbb{R}$, we have $\operatorname{Fix}(G)=\mathbb{R}$ and Proposition 3.3 implies generic linear growth regardless of the dynamics. 
Remark 3.6 By Field and Parry [6, Theorem 5.1.1], when $G$ is semisimple, the assumption of a connected hyperbolic attractor in Proposition 3.5(ii) may be weakened to the assumption that there is a hyperbolic basic set. In particular, this greater generality applies to $\mathbf{S E}(n), n \geq 3$, but not to $\mathbf{S E}(2)$.

\section{Extensions of chaotic base dynamics}

In this section we give answers to the question (Q2) asked in section 3. At present, our results are restricted to groups of the form $\Gamma=G \ltimes \mathbb{R}^{n}$ where either $G$ is abelian or $\operatorname{Fix}(\mathbf{T}) \neq 0$ for $\mathbf{T}$ is a maximal torus in $G$. In particular, this class of groups includes the Euclidean groups $\Gamma=\operatorname{SE}(n)$ when $n$ is odd and when $n=2$.

In subsection (a), we obtain some results of a topological nature on unboundedness of trajectories associated with a single $\omega$-limit set in the base. We prove unboundedness under certain assumptions on the group $\Gamma$ and on the number of periodic solutions in the $\omega$-limit set.

The results in subsection (a) do not apply directly to question (Q2) since the unboundedness could arise from $\bar{v}$ and/or from $\epsilon(N)$. The consequences of the results in subsection (a) for (Q2) are explored in subsection (b). As a special case, we obtain the result that if $G$ is abelian or if $\operatorname{Fix}(\mathbf{T}) \neq 0$, and if the base dynamics is "chaotic" then generically the deviation $\epsilon(N)$ from linear growth is unbounded.

\section{(a) Topological dynamics}

In this subsection, we consider the dynamics on $X$ restricted to a single $\omega$-limit set $A$. We state and prove the results only for maps. The results for flows are identical, with the convention that equilibria are counted as periodic solutions.

Definition 4.1 Suppose that $A \subset X$ is an $\omega$-limit set for the dynamics on $X$ and consider a $\Gamma$-extension $X \times \Gamma$ defined by some $\zeta: X \rightarrow \Gamma$. We say that a trajectory in $A \times \Gamma$ is unbounded if the closure of the trajectory is not compact. The dynamics on $A \times \Gamma$ is unbounded if there exists an unbounded trajectory in $A \times \Gamma$.

Suppose that the dynamics on $A \times \Gamma$ is unbounded in the sense of Definition 4.1. Then by continuity, for any $x_{0} \in X$ with $\omega\left(x_{0}\right)=A$ and for any $g_{0} \in G$, the component $v_{x_{0}, g_{0}}(N)$ of the trajectory with initial condition $\left(x_{0}, g_{0}, 0\right) \in X \times \Gamma$ is unbounded.

Proposition 4.2 Suppose that $\Gamma=G \ltimes \mathbb{R}^{n}$ with $G$ a compact connected Lie group, $\mathbf{T}$ a maximal torus in $G$ and $\operatorname{Fix}(\mathbf{T}) \neq 0$. Let $A \subset X$ be an $\omega$-limit set for the dynamics on $X$ and suppose that $A$ contains at least one periodic solution. Then for an open and dense set of $\Gamma$-extensions in the $C^{r}$ topology (for each $r \geq 0$ ), the dynamics on $A \times \Gamma$ is unbounded. 
Proof Let $P \subset A$ be a periodic solution. Since $\operatorname{Fix}(\mathbf{T}) \neq 0$, it follows from Proposition 3.1 (using the measure supported on $P$ ) that for generic extensions, trajectories on $P \times \Gamma$ are unbounded. Let $x$ be an initial condition in $X$ with $\omega(x)=A$. Then the trajectory of $(x, \gamma) \in X \times \Gamma$ limits on $P \times \Gamma$ for all $\gamma \in G$ and hence is unbounded.

Remark 4.3 The hypothesis that $\operatorname{Fix}(\mathbf{T}) \neq 0$ is satisfied trivially for the groups $\mathbb{R}^{n}$, and also for the Euclidean groups $\mathbf{S E}(n)$ when $n$ is odd.

Theorem 4.4 Suppose that $\Gamma=G \ltimes \mathbb{R}^{n}$ where $G$ is a compact connected abelian Lie group. Let $A \subset X$ be an w-limit set for the dynamics on $X$ and suppose that $A$ contains periodic solutions of arbitrarily high period. Then generically in the $C^{r}$ topology (for each $r \geq 0$ ) the dynamics on $A \times \Gamma$ is unbounded.

Proof The space $C^{r}(X, \Gamma)$ is a complete metric space. Let $Z_{j}=\left\{\zeta \in C^{r}(X, \Gamma)\right.$ : $\left|v_{x_{0}, e}(N)\right|>j$ for some $x_{0} \in X$, and some $\left.N \geq 1\right\}$. Each $Z_{j}$ is open. We now show that each $Z_{j}$ is dense and hence $\cap Z_{j}$ is the desired residual subset in $C^{r}(X, \Gamma)$. (In fact, our proof shows directly that $\cap Z_{j}$ is dense, without using the Baire Category Theorem.)

Let $U \times V \subset G \times \mathbb{R}^{n}$ be a neighborhood of the identity $(e, 0)$. We construct a cocycle using $\widetilde{\zeta}=(\widetilde{h}, \widetilde{k}): X \rightarrow U \times V$ such that $\widetilde{h}$ is constant, and such that $\widetilde{k}$, together with all of its derivatives, maps $X$ into $V$. Such a perturbation is arbitrarily small in the $C^{r}$ topology.

Since $G$ is a torus, the $N^{\prime}$ th roots of elements of $G$ are "equally spaced" in $G$. Hence there exists an integer $N_{0}$ such that every element in $G$ has an $N$ 'th root in $U$ for all $N \geq N_{0}$. Choose a periodic point $p \in A$ of minimal period $N \geq N_{0}$. Let $\left(p, h_{N}, k_{N}\right)=T^{N}(p, e, 0)$ and recall that by Proposition 2.1 we have

$$
\begin{aligned}
& h_{N}=h(p) h(f p) \cdots h\left(f^{N-1} p\right), \\
& k_{N}=k(p)+\rho_{h_{1}} k(f p)+\cdots+\rho_{h_{N-1}} k\left(f^{N-1} p\right) .
\end{aligned}
$$

In particular $h_{N}^{-1}$ has an $N^{\prime}$ th root $\tau \in U$. Since $G$ is abelian, $h(p) \tau \cdots h\left(f^{N-1} p\right) \tau=$ $h_{N} \tau^{N}=e \in G$. Hence, perturbing by the constant cocycle $\widetilde{h}(x) \equiv \tau, \widetilde{k}(x) \equiv 0$, we obtain that $h_{N}=e$.

Suppose after perturbation that $k_{N}=0$. Redefine $\widetilde{k}(x)$ so that $\widetilde{k}(p) \neq 0, \widetilde{k}(f p)=$ $\cdots=\widetilde{k}\left(f^{N-1} p\right)=0$. It is then the case that $k_{N} \neq 0$. Note that $\widetilde{k}(p)$ can be chosen arbitrarily small, and hence we can arrange that $\widetilde{k}$, together with all of its derivatives, maps $X$ into $V$.

We have shown that there is a small cocycle perturbation $(\widetilde{h}, \widetilde{k})$, of the form described above, after which we have $h_{N}=e, k_{N} \neq 0$. In particular, $T^{N \ell}(p, e, 0)=$ $\left(p, e, \ell k_{N}\right)$ and $\left|v_{p, e}(N \ell)\right|=\left|\ell k_{N}\right| \rightarrow \infty$ as $\ell \rightarrow \infty$ proving the theorem. 


\section{(b) Unboundedness of deviations}

Let $m$ be an ergodic measure on $X \times G$. Recall that the $\mathbb{R}^{n}$ component of a $\Gamma$-extension on $X \times \Gamma$ takes the form

$$
v_{x_{0}, g_{0}}(N)=\bar{v} N+\epsilon_{x_{0}, g_{0}}(N)
$$

with $\epsilon_{x_{0}, g_{0}}(N)=o(N)$ for $m$-almost all initial conditions $\left(x_{0}, g_{0}\right)$. Proposition 4.2 gives no information about whether the unboundedness of $v_{x_{0}, g_{0}}(N)$ arises from the term $\bar{v} N$ and/or the term $\epsilon_{x_{0}, g_{0}}(N)$. In fact, all three possibilities may occur: linear growth with bounded deviation (Proposition 3.1), unbounded sublinear growth (Theorem 4.5 below) and linear growth with unbounded deviation (also Theorem 4.5).

Theorem 4.5 Suppose that the ergodic measure $\mu$ on $X$ lifts to an ergodic measure $m=\mu \times \nu_{G}$ on $X \times G$ for a $C^{r}$ generic set of extensions $(r \geq 0)$. Let $\mathbf{T}$ denote a maximal torus in $G$. Suppose that at least one of the following conditions is satisfied:

(i) $\operatorname{Fix}(\mathbf{T}) \neq \operatorname{Fix}(G)$ and there is at least one periodic solution in the support of $\mu$.

(ii) $\operatorname{Fix}(\mathbf{T}) \neq 0$ and there are at least two periodic solutions in the support of $\mu$.

(iii) $G$ is abelian and there are infinitely many periodic solutions in the support of $\mu$.

Then generically, in the $C^{r}$ topology, $\epsilon_{x_{0}, g_{0}}(N)$ is unbounded for $\mu$-almost all $x_{0} \in X$ and for all $g_{0} \in G$.

In particular, under these hypotheses, generically we have unbounded sublinear growth if $\operatorname{Fix}(G)=\{0\}$ and linear growth with unbounded deviation if $\operatorname{Fix}(G) \neq\{0\}$.

Proof First, we prove part (i). Let $P$ denote a periodic solution in the support of $\mu$. Trajectories on $P \times \Gamma$ experience linear growth $\bar{v}_{P} \neq 0$ by Proposition 3.1. Moreover, $\bar{v}_{P}$ is a general element of $\operatorname{Fix}(\mathbf{T}) . \quad$ Write $\operatorname{Fix}(\mathbf{T})=\operatorname{Fix}(G) \oplus Y$, and define the projection $\pi: \mathbb{R}^{n} \rightarrow Y$. From the above discussion, $\pi \bar{v}_{P}$ is generically nonzero, so that the $Y$ components of trajectories on $P \times \Gamma$ are unbounded. As in Proposition 4.2, $\pi v_{x_{0}, g_{0}}(N)$ is unbounded for $\mu$-almost every $x_{0} \in X$ and for every $g_{0} \in G$. In addition, by ergodicity with respect to $m$, Proposition 3.4 implies that $\bar{v} \in \operatorname{Fix}(G)$ so that $\pi \bar{v}=0$. Hence $\pi \epsilon_{x_{0}, g_{0}}(N)$ is unbounded.

Next, we prove part (ii). Let $P_{1}$ and $P_{2}$ be distinct periodic solutions in the support of $\mu$. We have the three linear growth rates $\bar{v}, \bar{v}_{P_{1}}$ and $\bar{v}_{P_{2}}$ corresponding to the ergodic measure $m$ and the periodic solutions respectively. Since $\operatorname{Fix}(\mathbf{T}) \neq 0$, generically $\bar{v}_{P_{1}}$ and $\bar{v}_{P_{2}}$ are nonzero. Moreover, generically $\bar{v}_{P_{1}} \neq \bar{v}_{P_{2}}$. 
Now we pass to a traveling frame with speed $\bar{v}$. More precisely, we replace the $\Gamma$-extension $(h, k)$ by the new extension $\left(h^{\prime}, k^{\prime}\right)=(h, k-\bar{v})$. Note that trajectories for the extension $\left(h^{\prime}, k^{\prime}\right)$ satisfy

$$
v_{x_{0}, g_{0}}^{\prime}(N)=\bar{v} N+\epsilon_{x_{0}, g_{0}}(N)-\bar{v} N=\epsilon_{x_{0}, g_{0}}(N),
$$

so it remains to show unboundedness for the extension $\left(h^{\prime}, k^{\prime}\right)$.

For the extension $\left(h^{\prime}, k^{\prime}\right)$, the periodic solutions have corresponding linear growth rates $\bar{v}_{P_{j}}^{\prime}=\bar{v}_{P_{j}}-\bar{v}$. Since $\bar{v}_{P_{1}} \neq \bar{v}_{P_{2}}$, at least one of the growth rates $\bar{v}_{P_{j}}^{\prime}$ is nonzero. Hence trajectories for the extension $\left(h^{\prime}, k^{\prime}\right)$ are unbounded as required.

Finally, we prove part (iii). If $\operatorname{Fix}(G) \neq 0$ then $\operatorname{Fix}(\mathbf{T}) \neq 0$ and we can apply part (ii). If $\operatorname{Fix}(G)=\{0\}$ then $\bar{v}=0$ by Proposition 3.4, and $v(N)$ is unbounded by Theorem 4.4, so that $\epsilon(N)$ is unbounded.

Remark 4.6 (a) The Euclidean groups $\Gamma=\mathrm{SE}(n)$ satisfy certain of the hypotheses of Theorem 4.5 when $n$ is odd and when $n=2$. In particular, if periodic points are dense in the support of $\mu$, we have unbounded sublinear growth generically for the Euclidean groups with $n \geq 3$ odd and with $n=2$. (The case $\mathbf{S E}(1)$ is a special case of $\Gamma=\mathbb{R}^{n}, n \geq 1$, for which we have linear growth with unbounded deviation generically.)

(b) Under hyperbolicity assumptions on the dynamics on $X$, it can be shown that the conclusion of Theorem 4.5 is valid for all compact connected Lie groups $G$, see Section 6. In fact, we conjecture that part (iii) of Theorem 4.5 is valid even if $G$ is not abelian and without hyperbolicity assumptions.

\section{$5 \quad$ Equivariant deterministic central limit theorems}

Throughout this section, $X$ is a compact Riemannian manifold and the base dynamics $f: X \rightarrow X$ is a topologically mixing Axiom A diffeomorphism when restricted to a hyperbolic basic set equipped with a Gibbs measure $\mu$. Let $G$ be a compact Lie group with Haar measure $\nu_{G}$ and define the product measure $m=\mu \times \nu_{G}$ on $X \times G$. We consider $G$-extensions $S: X \times G \rightarrow X \times G$.

Most of the results in this section require additional hypotheses on the base dynamics $f$ or on the group $G$. We define hypothesis $(\mathrm{H})$ by

(H) The Axiom A diffeomorphism $f$ is Anosov on an infranilmanifold, and/or the compact Lie group $G$ is semisimple.

Let $\rho$ be a representation of $G$ on $\mathbb{R}^{n}$ and suppose that $\Phi: X \times \Gamma \rightarrow \mathbb{R}^{n}$ is a smooth function with mean zero. We prove an equivariant deterministic central limit 
theorem which states that if hypothesis $(\mathrm{H})$ holds and $\Phi$ is equivariant (as defined in subsection (a)), then generically $\frac{1}{\sqrt{N}} \sum_{j=0}^{N-1} \Phi \circ S^{j}$ converges in distribution to an $n$-dimensional normal distribution. The normal distribution is equivariant (again defined in subsection (a)) and the form of the normal distribution (general, standard, and so on) depends on the representation of $G$. Moreover, we give necessary and sufficient conditions for nondegeneracy of this normal distribution in terms of the unboundedness of the sum $\sum_{j=0}^{N-1} \Phi \circ S^{j}$.

In subsection (a), we begin by stating a recent result of Liverani [10] that establishes conditions for a one-dimensional central limit theorem on $X \times G$. We then prove an $n$-dimensional equivariant version of this result.

The condition for nondegeneracy in the central limit theorems of subsection (a) takes the form of a cohomological equation. Livšic type theorems state that measurable solutions to cohomological equations have Hölder continuous versions. In subsection (b), we prove an equivariant Livšic theorem and use this to investigate the solutions of the cohomological equation.

In subsection (c), we state our main result, namely that provided hypothesis $(\mathrm{H})$ is satisfied, then generically $\frac{1}{\sqrt{N}} \sum_{j=0}^{N-1} \Phi \circ S^{j}$ converges in distribution to a nondegenerate equivariant $n$-dimensional normal distribution. The proof relies on the results of subsection (a) and (b) together with recent results of Dolgopyat [5], Burns and Wilkinson [4], and Field and Parry [6].

In subsection (d), we specialize our results to the cases $G=\mathbf{1}, n \geq 1$, and $G=\mathbf{S O}(n), n \geq 2$. In particular, in the case $G=\mathbf{S O}(n)$, generically we obtain convergence to a nondegenerate standard normal distribution. In contrast, in the case $G=\mathbf{1}$, generically the covariance matrix has nonzero offdiagonal entries.

\section{(a) An $n$-dimensional equivariant central limit theorem}

Let $X$ be a compact Riemannian manifold and suppose that the base dynamics $f: X \rightarrow X$ is a topologically mixing Axiom A diffeomorphism when restricted to a hyperbolic basic set equipped with a Gibbs measure $\mu$. Let $G$ be a compact connected Lie group and define the product measure $m=\mu \times \nu_{G}$ on $X \times G$. We consider $G$-extensions $S: X \times G \rightarrow X \times G$ given by $S(x, g)=(f(x), g h(x))$ with $h \in C^{r}(X, G)$.

In this subsection, we assume that $S: X \times G \rightarrow X \times G$ is rapidly mixing. That is, for any $k \geq 1$, and for all $C^{\infty}$ functions $\phi, \psi: X \times G \rightarrow \mathbb{R}$, there is a constant $C>0$ such that

$$
\left|\int_{X \times G} \phi(y) \psi\left(S^{j}(y)\right) d m(y)-\int_{X \times G} \phi d m \int_{X \times G} \psi d m\right| \leq C j^{-k},
$$

for all $j \geq 1$. This assumption turns out to be valid generically provided hypothesis $(\mathrm{H})$ is satisfied by results of $[4,5,6]$, see subsection $(\mathrm{c})$. 
We start by stating a recent theorem of Liverani.

Theorem 5.1 (Liverani [10, Theorem 1.2]) Suppose that $f: X \rightarrow X$ is $A x$ iom $A$ and suppose that $S: X \times G \rightarrow X \times G$ is rapidly mixing. Let $\phi: X \times G \rightarrow \mathbb{R}$ be a $C^{\infty}$ function with mean zero $\left(\int_{X \times G} \phi d m=0\right)$. Then, $S$ satisfies a central limit theorem for $\phi$. That is, there exists $\sigma \geq 0$ such that for each interval $I \subset \mathbb{R}$,

$$
\lim _{N \rightarrow \infty} m\left\{y \in X \times G: \frac{1}{\sqrt{N}} \sum_{j=0}^{N-1} \phi\left(S^{j} y\right) \in I\right\}=\frac{1}{\left(2 \pi \sigma^{2}\right)^{1 / 2}} \int_{I} e^{-x^{2} / 2 \sigma^{2}} d x
$$

Moreover, $\sigma=0$ if and only if the cohomological equation

$$
\phi(y)=\psi(S y)-\psi(y),
$$

has an $L^{2}$ solution $\psi: X \times G \rightarrow \mathbb{R}$.

Remark 5.2 (a) Theorem 5.1 is a special case of [10, Theorem 1.2] also stated explicitly in Dolgopyat [5, Corollary 6.1].

(b) There is an extensive theory concerning central limit theorems for Axiom A dynamical systems and their generalizations, see Young [17] and references therein.

Theorem 5.1 gives conditions under which a sequence of random variables $X_{N}$ : $X \times G \rightarrow \mathbb{R}$ (specifically $X_{N}=\frac{1}{\sqrt{N}} \sum_{j=0}^{N-1} \phi \circ S^{j}$ ) converges in distribution to a normal distribution with mean zero and variance $\sigma^{2}$. Moreover, the theorem gives necessary and sufficient conditions for the normal distribution to be nondegenerate $(\sigma>0)$.

In this subsection, we generalize Liverani's Theorem in two directions. First, we prove the $n$-dimensional analogue, where $\phi$ takes values in $\mathbb{R}^{n}$. Second, we prove an equivariant analogue.

Suppose that $X$ is a random vector taking values in $\mathbb{R}^{n}$. Recall that $X$ is said to have an $n$-dimensional normal distribution with mean zero if the distribution function of $X$ is given by

$$
\frac{1}{(2 \pi)^{n / 2}(\operatorname{det} \Sigma)^{1 / 2}} \int_{I} \exp \left\{-\frac{1}{2}\left\langle\Sigma^{-1} x, x\right\rangle\right\} d x_{1} \cdots d x_{n},
$$

where $\Sigma$ is an $n \times n$ matrix, called the covariance matrix. It is easily verified that $\Sigma_{j k}=E\left(X_{j} X_{k}\right)$, so that $\Sigma$ is symmetric and $\langle\Sigma x, x\rangle \geq 0$ for all $x \in \mathbb{R}^{n}$. The distribution is said to be nondegenerate if $\Sigma$ is nonsingular, (equivalently, $\langle\Sigma x, x\rangle>0$ for all nonzero $\left.x \in \mathbb{R}^{n}\right){ }^{2}$

The Cramer-Wold technique (see for example [3, Theorem 29.4]) enables us to pass from 1-dimensional normal distributions to $n$-dimensional normal distributions:

\footnotetext{
${ }^{2}$ In the degenerate case $\operatorname{det} \Sigma=0$, the singular density function defines in the obvious way a normal distribution concentrated on a proper subspace of $\mathbb{R}^{n}$.
} 
Lemma 5.3 Let $X(N)=\left(X_{1}(N), \cdots, X_{n}(N)\right)$ be a sequence of random vectors with values in $\mathbb{R}^{n}$. Suppose that for any $\alpha=\left(\alpha_{1}, \ldots, \alpha_{n}\right) \in \mathbb{R}^{n}$, the sequence $\sum_{j=1}^{n} \alpha_{j} X_{j}(N)$ converges in distribution to a (possibly degenerate) normal distribution with mean zero. Then $X(N)$ converges in distribution to a (possibly degenerate) $n$-dimensional normal distribution with mean zero and covariance matrix $\Sigma=\left\{E\left(X_{j} X_{k}\right)\right\}_{1 \leq j, k \leq n}$.

Moreover, if the sequence $\sum_{j=1}^{n} \alpha_{j} X_{j}(N)$ converges to a nondegenerate normal distribution for all $\alpha \neq 0$, then $X(N)$ converges in distribution to a nondegenerate n-dimensional normal distribution.

Let $\rho$ denote a representation of $G$ on $\mathbb{R}^{n}$. A map $\Phi: X \times G \rightarrow \mathbb{R}^{n}$ is $G$-equivariant if $\Phi(x, a g)=\rho_{a} \Phi(x, g)$ for all $a \in G$. (Equivalently, $\Phi(x, g)=\rho_{g} k(x)$ for some map $k: X \rightarrow \mathbb{R}^{n}$.)

Similarly, a linear map $\Sigma: \mathbb{R}^{n} \rightarrow \mathbb{R}^{n}$ is $G$-equivariant if $\rho_{g} \Sigma=\Sigma \rho_{g}$ for all $g \in G$. Finally, we say that an $n$-dimensional normal distribution is $G$-equivariant if the covariance matrix $\Sigma$ is $G$-equivariant.

Theorem 5.4 Suppose that $f: X \rightarrow X$ is Axiom $A$ and suppose that $S: X \times G \rightarrow$ $X \times G$ is rapidly mixing. Let $\Phi: X \times G \rightarrow \mathbb{R}^{n}$ be a $C^{\infty} G$-equivariant function with mean zero. Then $\frac{1}{\sqrt{N}} \sum_{j=0}^{N-1} \Phi \circ S^{j}$ converges in distribution to a (possibly degenerate) $G$-equivariant $n$-dimensional normal distribution with mean zero. That is, there is a $G$-equivariant $n \times n$ matrix $\Sigma$ such that for each cube $I \subset \mathbb{R}^{n}$,

$\lim _{N \rightarrow \infty} m\left\{y \in X \times G: \frac{1}{\sqrt{N}} \sum_{j=0}^{N-1} \Phi\left(S^{j} y\right) \in I\right\}=\frac{1}{(2 \pi)^{n / 2}(\operatorname{det} \Sigma)^{1 / 2}} \int_{I} \exp \left\{-\frac{1}{2}\left\langle\Sigma^{-1} x, x\right\rangle\right\} d x$.

The distribution is degenerate if and only if there is a $G$-invariant subspace $V \subset$ $\mathbb{R}^{n}$, with associated orthogonal projection $\pi_{V}: \mathbb{R}^{n} \rightarrow V$, such that the restricted cohomological equation $\pi_{V} \Phi=\Psi \circ S-\Psi$ has a $G$-equivariant $L^{2}$ solution $\Psi: X \times G \rightarrow V$.

Proof We introduce the sequence of random vectors $X(N): X \times G \rightarrow \mathbb{R}^{n}$ given by $X(N)=\frac{1}{\sqrt{N}} \sum_{j=0}^{N-1} \Phi \circ S^{j}$. Let $\pi: \mathbb{R}^{n} \rightarrow \mathbb{R}$ be a linear function, and consider the $C^{\infty}$ function $\phi=\pi \Phi: X \times G \rightarrow \mathbb{R}$. Then $\phi$ has mean zero and it follows from Theorem 5.1 that the sequence of random variables $\pi X(N)$ converges in distribution to a normal distribution with mean zero. Since the projection $\pi$ is arbitrary, it follows from Lemma 5.3 that $X(N)$ converges in distribution to an $n$-dimensional normal distribution with mean zero and covariance matrix $\Sigma$.

If $\Phi$ is $G$-equivariant then $X(N)$ is $G$-equivariant and $X(N)(x, a g)=\rho_{a} X(N)(x, g)$ for all $a \in G$. By left invariance of Haar measure,

$$
m\{(x, g) \in X \times G: X(N)(x, a g) \in I\}=m\{(x, g) \in X \times G: X(N)(x, g) \in I\} .
$$


It follows that $X(N)$ and $\rho_{a} X(N)$ converge to the same normal distribution. We compute, making a change of variables, that for each $a \in G$,

$$
\begin{array}{r}
\frac{1}{(\operatorname{det} \Sigma)^{1 / 2}} \int_{I} \exp \left\{-\frac{1}{2}\left\langle\Sigma^{-1} x, x\right\rangle\right\} d x=\frac{1}{(\operatorname{det} \Sigma)^{1 / 2}} \int_{\rho_{a}^{-1} I} \exp \left\{-\frac{1}{2}\left\langle\Sigma^{-1} x, x\right\rangle\right\} d x \\
=\frac{1}{\left(\operatorname{det}\left(\rho_{a} \Sigma \rho_{A}^{-1}\right)\right)^{1 / 2}} \int_{I} \exp \left\{-\frac{1}{2}\left\langle\left(\rho_{a} \Sigma \rho_{a}^{-1}\right)^{-1} x, x\right\rangle\right\} d x .
\end{array}
$$

It follows that $\rho_{a} \Sigma \rho_{a}^{-1}=\Sigma$ for each $a \in G$ so that $\Sigma$ is $G$-equivariant.

It is immediate from the last statements of Theorem 5.1 and Lemma 5.3 that the $n$-dimensional normal distribution is degenerate if and only if there is a projection $\pi: \mathbb{R}^{n} \rightarrow \mathbb{R}$ such that $\pi \Phi=\psi \circ S-\psi$ has an $L^{2}$ solution $\psi$. In particular, it is immediate that the distribution is degenerate if $\pi_{V} \Phi=\Psi \circ S-\Psi$ has an $L^{2}$ solution $\Psi$ for some $G$-invariant subspace $V$.

Conversely, suppose that the $n$-dimensional normal distribution is degenerate. Then there is a projection $\pi$ and an $L^{2}$ function $\psi: X \times G \rightarrow \mathbb{R}$ such that $\pi \Phi=\psi \circ S-$ $\psi$. For each $g \in G$ we can form the projection $\pi \rho_{g}: V \rightarrow \mathbb{R}$. Let $K=\cap_{g \in G} \operatorname{ker} \pi \rho_{g}$. Then $K$ is $G$-invariant and has a $G$-invariant complement $V$ of dimension $r$ say. Choose group elements $g_{1}, \ldots, g_{r} \in G$ with $g_{1}=e$, such that the projections $\pi_{j}=$ $\pi \rho_{g_{j}}: V \rightarrow \mathbb{R}$ are linearly independent. That is, $\pi_{j} v=0$ for all $j$ if and only if $v=0$. In addition, given $v_{1}, \ldots, v_{r} \in \mathbb{R}$, there exists (a unique) $v \in V$ such that $\pi_{j} v=v_{j}$. Let $\pi_{V}: \mathbb{R}^{n} \rightarrow V$ denote the orthogonal projection. Observe that $\pi_{V} \Phi$ is $G$-equivariant. Define $\Phi_{j}=\pi_{j} \Phi_{V}$ and compute that $\Phi_{j}(x, g)=\phi\left(x, g_{j} g\right)$. Define $\Psi_{j}(x, g)=\psi\left(x, g_{j} g\right)$. By definition, $\Phi_{1}=\Psi_{1} \circ S-\Psi_{1}$ and it follows from equivariance of $S$ and $\Phi_{V}$ that $\Phi_{j}=\Psi_{j} \circ S-\Psi_{j}$ for all $j$. Thus the measurable function $\Psi: X \times G \rightarrow V$ with components $\pi_{j} \Psi=\Psi_{j}$ satisfies the restricted cohomological equation $\pi_{V} \Phi=\Psi \circ S-\Psi$. Averaging over $G$ yields a $G$-equivariant solution $\Psi$.

Remark 5.5 It follows from the theorem that we expect convergence to a standard normal distribution (where the covariance matrix is a real multiple of the identity) if and only if the action $\rho$ of $G$ on $\mathbb{R}^{n}$ is irreducible.

\section{(b) An equivariant Livšic Theorem}

In this subsection, we continue to suppose that $f: X \rightarrow X$ is Axiom A when restricted to a hyperbolic basic set $\Lambda \subset X$ equipped with a Gibbs measure $\mu$. Again, we suppose that $G$ is a compact Lie group and define $m=\mu \times \nu_{G}$. We consider smooth $G$-extensions $S: X \times G \rightarrow X \times G$.

Let $V$ be a finite dimensional vector space, and let $\rho$ denote a representation of $G$ on $V$. Again, we say that a map $\Phi: X \times G \rightarrow V$ is $G$-equivariant if $\Phi(x, a g)=$ $\rho_{a} \Phi(x, g)$ for all $a \in G$. 
Theorem 5.6 Suppose that the G-extension $S: X \times G \rightarrow X \times G$ is such that $m=$ $\mu \times \nu_{G}$ is ergodic. Let $\Phi: \Lambda \times G \rightarrow V$ be a $G$-equivariant Hölder continuous function. Then any $G$-equivariant measurable solution $\Psi: \Lambda \times G \rightarrow V$ to the cohomological equation

$$
\Phi=\Psi \circ S-\Psi
$$

has a Hölder continuous version. That is, there exists a Hölder continuous function $\Psi^{\prime}: \Lambda \times G \rightarrow \mathbb{R}$ such that $\Psi^{\prime}=\Psi$ almost everywhere.

Proof The proof combines the original argument of Livšic [11] with recent results on partially hyperbolic sets [13].

Step 1 In [13, Theorem 4.1], local stable manifolds $W^{s}(x, g) \subset X \times G$ are constructed for each $(x, g) \in \Lambda \times G$ as Hölder continuous graphs over the local stable manifolds $W^{s}(x) \subset X$. Moreover, the Hölder exponents and constants are uniform in $x$ and $g$. The same is true of the local unstable manifolds $W^{u}(x, g)$. (We note that the partial hyperbolicity assumption in [13] is automatically satisfied since $G$ is compact.)

Step 2 Choose a metric $d_{X}$ on $X$ and a right-invariant metric $d_{G}$ on $G$. Define the right-invariant metric $d$ on $X \times G$ by setting $d\left(\left(x_{1}, g_{1}\right),\left(x_{2}, g_{2}\right)\right)=d_{X}\left(x_{1}, x_{2}\right)+$ $d_{G}\left(g_{1}, g_{2}\right)$. It follows easily from the local product structure on $\Lambda$ together with the uniform Hölder continuity of the local manifolds $W^{s}(x, g), W^{u}(x, g)$ that we have the following analogue of local product structure on $\Lambda \times G$. There exists $\epsilon>0$ such that if $\left(x_{1}, g_{1}\right),\left(x_{2}, g_{2}\right) \in \Lambda \times G$ satisfy $d\left(\left(x_{1}, g_{1}\right),\left(x_{2}, g_{2}\right)\right)<\epsilon$ then $W^{u}\left(x_{1}, g_{1}\right) \cap$ $W^{s}\left(x_{2}, b g_{2}\right) \cap \Lambda \times G \neq \emptyset$ for some $b \in G$. For notational convenience, we scale $d_{G}$ so that $d_{G}(b, e)<1$ for all $b \in G$.

Step 3 It follows from the original argument of Livšic that $\Psi$ is Hölder continuous on $W^{s}(x, g)$ and $W^{u}(x, g)$ for almost every $(x, g) \in \Lambda \times G$. In fact, it follows from equivariance of $\Psi$ that Hölder continuity holds on $W^{s}(x, g)$ and $W^{u}(x, g)$ for almost every $x \in \Lambda$ and for every $g \in G$. As usual, the Hölder exponent $\alpha>0$ and constant $K>0$ are uniform in $(x, g)$.

Step 4 Let $\epsilon>0$ be as in Step 2, and suppose that $d\left(\left(x_{1}, g_{1}\right),\left(x_{2}, g_{2}\right)\right)<\epsilon$. By Step 2, we may choose $(x, g) \in \Lambda \times G$ and $b \in G$ such that $(x, g) \in W^{u}\left(x_{1}, g_{1}\right) \cap$ $W^{s}\left(x_{2}, b g_{2}\right)$. By ergodicity on $\Lambda \times G$ together with the absolute continuity of the foliations $\left\{W^{s}(x, g)\right\},\left\{W^{u}(x, g)\right\}$ [13], for almost every $x_{1}, x_{2}$ and for all $g_{1}, g_{2}$, we have

$$
\begin{gathered}
\left|\Psi\left(x_{1}, g_{1}\right)-\Psi(x, g)\right| \leq K d\left(\left(x_{1}, g_{1}\right),(x, g)\right)^{\alpha} \\
\left|\Psi(x, g)-\Psi\left(x_{2}, b g_{2}\right)\right| \leq K d\left((x, g),\left(x_{2}, b g_{2}\right)\right)^{\alpha},
\end{gathered}
$$


by Step 3. (Again, $\alpha$ and $K$ are absolute constants.) In addition, there is a Lipschitz constant $K_{1}$ such that

$$
\left\|\rho_{b}-I\right\| \leq K_{1} d_{G}(b, e) \leq K_{1} d_{G}(b, e)^{\alpha}=K_{1} d_{G}\left(b g_{2}, g_{2}\right)^{\alpha}=K_{1} d\left(\left(x_{2}, b g_{2}\right),\left(x_{2}, g_{2}\right)\right)^{\alpha},
$$

for all $\left(x_{2}, g_{2}\right) \in \Lambda \times G$, and for all $b \in G$. Hence, it follows from equivariance of $\Psi$ that

$$
\left|\Psi\left(x_{2}, b g_{2}\right)-\Psi\left(x_{2}, g_{2}\right)\right|=\left|\left(\rho_{b}-I\right) \Psi\left(x_{2}, g_{2}\right)\right| \leq K_{1}\left|\Psi\left(x_{2}, g_{2}\right)\right| d\left(\left(x_{2}, b g_{2}\right),\left(x_{2}, g_{2}\right)\right)^{\alpha}
$$

Together, inequalities $(5.3,5.4,5.5)$ imply that there are absolute constants $\epsilon>0$, $\alpha>0$ and $K_{2}>0$ such that

$$
\left|\Psi\left(x_{1}, g_{1}\right)-\Psi\left(x_{2}, g_{2}\right)\right| \leq K_{2} d\left(\left(x_{1}, g_{1}\right),\left(x_{2}, g_{2}\right)\right)^{\alpha},
$$

for almost all $\left(x_{1}, g_{1}\right),\left(x_{2}, g_{2}\right)$ that are $\epsilon$-close. In this way, we obtain uniform Hölder continuity in an $\epsilon$-neighborhood of $\left(x_{2}, g_{2}\right)$ for almost all $\left(x_{2}, g_{2}\right)$ and hence Hölder continuity on the whole of $\Lambda \times G$.

We are now able to give precise conditions that determine the degeneracy or nondegeneracy of the normal distribution in Theorem 5.4.

Corollary 5.7 Assume the hypotheses and conclusions of Theorem 5.4. The following are equivalent:

(i) The normal distribution is degenerate.

(ii) There is a $G$-invariant subspace $V \subset \mathbb{R}^{n}$ such that the sequence $\pi_{V} \sum_{j=0}^{N-1} \Phi\left(S^{j}(y)\right)$ is bounded uniformly in $y \in \Lambda \times G$ and $N \geq 1$.

(iii) There is a $G$-invariant subspace $V \subset \mathbb{R}^{n}$ such that the cohomological equation $\pi_{V} \Phi=\Psi \circ S-\Psi$ has a Hölder solution $\Psi$.

(In cases (ii) and (iii), the covariance matrix $\Sigma$ satisfies $\left.\Sigma\right|_{V}=0$ ).

Proof Statements (i) and (iii) are equivalent by Theorems 5.4 and 5.6. If $\pi_{V} \sum_{j=0}^{N-1} \Phi \circ$ $S^{j}$ is bounded, then it is immediate that $\frac{1}{\sqrt{N}} \pi_{V} \sum_{j=0}^{N-1} \Phi \circ S^{j}$ converges to the degenerate distribution supported at 0 showing that (ii) implies (i). Conversely, suppose that $\pi_{V} \Phi=\Psi \circ S-\Psi$ has a Hölder solution $\Psi: X \times G \rightarrow \mathbb{R}$. In particular, $\pi_{V} \sum_{j=0}^{N-1} \Phi \circ S^{j}=\Psi \circ S^{N}-\Psi$. Since $\Lambda \times G$ is compact and $\Psi$ is continuous, it follows that the sequence $\pi_{V} \sum_{j=0}^{N-1} \Phi \circ S^{j}$ is bounded, so (iii) implies (ii). 
Write $S(x, g)=(f(x), g h(x)), \pi_{V} \Phi(x, g)=\rho_{g} k(x)$, and $\Psi(x, g)=\rho_{g} \ell(x)$. The restricted cohomological equation $\pi_{V} \Phi=\Psi \circ S-\Psi$ reduces to the equation

$$
k(x)=\rho_{h(x)} \ell(f x)-\ell(x) .
$$

The next result shows that typically this equation has no continuous solutions.

Lemma 5.8 Let $X$ be a topological space, $G$ be a compact topological group, and $\rho: G \rightarrow L\left(\mathbb{R}^{n}\right)$ be an orthogonal representation of $G$. Let $h: X \rightarrow G$ be continuous. Suppose that $f: X \rightarrow X$ is a homeomorphism with a fixed point $x_{0} \in X$, and that there is a "homoclinic point" $x \in X$ with the property that $x \neq x_{0}$ and $f^{N}(x) \rightarrow x_{0}$ as $N \rightarrow \pm \infty$.

Then there is an open and dense set of functions $k: X \rightarrow \mathbb{R}^{n}$ in the $C^{r}$ topology (for each $r \geq 0$ ) such that the cohomological equation (5.6) has no continuous solutions $\ell: X \rightarrow \mathbb{R}^{n}$.

Proof Let $A=\rho_{h\left(x_{0}\right)}-I$. The proof divides into two cases: $A$ singular and $A$ nonsingular. First, we show that equation (5.6) has no solutions unless $k\left(x_{0}\right)$ lies in the range of $A$, which deals with the case $A$ singular. In the case when $A$ is nonsingular, we use the fact that $x$ is homoclinic to choose a neighborhood $U$ of $x$ such that $f^{j}(x) \notin U$ for all $|j| \geq 1$. The value of $k(x)$ can be changed slightly by a $C^{r}$ small perturbation supported in $U$ and we show that equation (5.6) has no continuous solutions for every perturbation of this form.

Suppose that $\ell: X \rightarrow \mathbb{R}^{n}$ is a continuous solution to equation (5.6). Then in particular $k\left(x_{0}\right)=A \ell\left(x_{0}\right)$, so that $k\left(x_{0}\right)$ lies in the range of $A$ as claimed above. Hence, we may suppose from now on that $A$ is nonsingular, so that $\ell\left(x_{0}\right)=A^{-1} k\left(x_{0}\right)$.

Now we use the homoclinic point $x$. Define $h_{j} \in G$ as follows:

$$
h_{j}= \begin{cases}h(x) h(f x) \cdots h\left(f^{j-1} x\right) ; & j \geq 1 \\ h\left(f^{-1} x\right)^{-1} h\left(f^{-2} x\right)^{-1} \cdots h\left(f^{-j}\right)^{-1} ; & j \leq-1\end{cases}
$$

and $h_{0}=e$. It is easily verified that

$$
h_{j} h\left(f^{j} x\right)=h_{j+1},
$$

for all $j \in \mathbb{Z}$. Using equations (5.6) and (5.7), we compute that

$$
\begin{aligned}
& \sum_{j=-N}^{N} \rho_{h_{j}} k\left(f^{j} x\right)=\sum_{j=-N}^{N} \rho_{h_{j}}\left(\rho_{h\left(f^{j} x\right)} \ell\left(f^{j+1} x\right)-\ell\left(f^{j} x\right)\right) \\
& \quad=\sum_{j=-N}^{N} \rho_{h_{j+1}} \ell\left(f^{j+1} x\right)-\rho_{h_{j}} \ell\left(f^{j} x\right)=\rho_{h_{N+1}} \ell\left(f^{N+2} x\right)-\rho_{h_{-N}} \ell\left(f^{-N} x\right) .
\end{aligned}
$$


Passing to a subsequence, we may suppose that $h_{N} \rightarrow h^{ \pm}$as $N \rightarrow \pm \infty$. Then

$$
\sum_{j=-N}^{N} \rho_{h_{j}} k\left(f^{j} x\right) \rightarrow\left(\rho_{h^{+}}-\rho_{h^{-}}\right) \ell\left(x_{0}\right)=\left(\rho_{h^{+}}-\rho_{h^{-}}\right) A^{-1} k\left(x_{0}\right) .
$$

Changing the value of $k(x)$ by a perturbation supported in $U$, as described above, leads to a corresponding change in the limit of the left-hand-side expression yielding the required contradiction.

\section{(c) Nondegenerate central limit theorems}

We continue to suppose that $f: X \rightarrow X$ is a topologically mixing Axiom A diffeomorphism when restricted to a hyperbolic basic set $\Lambda \subset X$ equipped with a Gibbs measure $\mu$, and that $\mathbb{R}^{n}$ is a representation of a compact connected Lie group $G$. Suppose that $S: X \times G \rightarrow X \times G$ is a rapidly mixing $G$-extension of $f$ and that $\Phi: X \times G \rightarrow \mathbb{R}$ is a $C^{\infty}$ function with mean zero. We proved in Theorem 5.4 that $\frac{1}{\sqrt{N}} \sum_{j=0}^{N-1} \Phi \circ S^{j}$ converges in distribution to a $G$-equivariant $n$-dimensional normal distribution with mean zero.

To apply Theorem 5.4, we need hypotheses that guarantee that $S$ is rapidly mixing. It turns out that $S$ is generically rapidly mixing provided we assume hypothesis $(H)$ stated at the beginning of this section. Sufficiency of hypothesis $(H)$ is shown by the following recent results of Field and Parry [6] and Dolgopyat [5].

Theorem 5.9 (Field and Parry [6, Theorem 5.1.1]) Suppose that hypothesis (H) is satisfied. (For alternative assumptions on $f$, see [6].) Suppose that $S: X \times G \rightarrow$ $X \times G$ is $C^{r}$ where $r \geq 1$. Then generically $S$ is stably ergodic. That is, the subset of $C^{r}$ extensions $h: X \rightarrow G$ for which $m=\mu \times \nu_{G}$ is ergodic is open and dense in the $C^{r}$ topology.

Theorem 5.10 (Dolgopyat [5, Corollaries 1.2 and 1.3]) Suppose that hypothesis (H) is satisfied. If $S: X \times G \rightarrow X \times G$ is a stably ergodic extension of $f$ then $S$ is rapidly mixing.

Theorem 5.11 Suppose that $f: X \rightarrow X$ is a topologically mixing Axiom $A$ diffeomorphism, and let $\mathbb{R}^{n}$ be a representation of a compact connected Lie group $G$. Suppose that hypothesis (H) is satisfied. Then there is an open and dense set of $G$ extensions $S: X \times G \rightarrow X \times G$ and mean zero $G$-equivariant functions $\Phi: X \times G \rightarrow \mathbb{R}^{n}$ in the $C^{r}$ topology (for each $r \geq 1$ ) such that $\frac{1}{\sqrt{N}} \sum_{j=0}^{N-1} \Phi \circ S^{j}$ converges in distribution to a nondegenerate $G$-equivariant $n$-dimensional normal distribution with mean zero. 
Moreover, the distribution is a general nondegenerate G-equivariant normal distribution. More precisely, if $\Sigma(\Phi)$ is the covariance matrix associated with a $G$ equivariant map $\Phi$, then the mapping $\Phi \mapsto \Sigma(\Phi)$ is an open mapping onto the set of $G$-equivariant positive definite symmetric matrices.

Proof By Theorems 5.9 and 5.10, generically the corresponding $G$-extension $S: X \times$ $G \rightarrow X \times G$ is rapidly mixing. Hence, by Theorem 5.4, $\frac{1}{\sqrt{N}} \sum_{j=0}^{N-1} \Phi \circ S^{j}$ converges in distribution to a $G$-equivariant $n$-dimensional normal distribution with mean zero. By Lemma 5.8, generically the cohomological equation (5.6) has no continuous solutions, guaranteeing nondegeneracy of the normal distribution by Corollary 5.7.

It remains to show that $\Sigma$ is a general $G$-equivariant symmetric positive definite matrix. A computation using change of variables (similar to the proof of equivariance of $\Sigma$ in the proof of Theorem 5.4) shows that if $A$ is an $n \times n$ matrix then the sequence $\frac{1}{\sqrt{N}} \sum_{j=0}^{N-1} A \Phi \circ S^{j}$ converges in distribution to the normal distribution with mean zero and covariance matrix $A \Sigma A^{T}$. In particular, in the absence of symmetry, the result follows from the fact that all positive definite symmetric matrices are congruent. When symmetry is present, we require some elementary representation theory (over $\mathbb{R}$ ). First, write $\mathbb{R}^{n}=W_{1} \oplus \cdots \oplus W_{r}$ where $W_{1}, \ldots, W_{r}$ are the isotypic components for the action of $G$ (so each $W_{j}$ is a direct sum of isomorphic irreducible representations, and distinct $W_{j}$ 's consist of distinct irreducible representations). It is immediate that $\Sigma=\Sigma_{1} \oplus \cdots \oplus \Sigma_{r}$ where $\Sigma_{j}: W_{j} \rightarrow W_{j}$. Hence, we may suppose without loss of generality that $\mathbb{R}^{n}$ is a single isotypic component. Second, write $\mathbb{R}^{n}=V \oplus \cdots \oplus V$ ( $s$ copies) where $V$ is an irreducible representation of $G$. The real version of Schur's Lemma states that the space of $G$-equivariant linear maps on $V$ is a real division ring $\mathcal{D}$ isomorphic to $\mathbb{R}, \mathbb{C}$ or $\mathbb{H}$. Hence $\Sigma($ and $A$ ) is identified with an $s \times s$ matrix with entries in $\mathcal{D}$. When $\mathcal{D}=\mathbb{R}$, we have reduced to the case without symmetry, and the cases $\mathcal{D}=\mathbb{C}$ and $\mathcal{D}=\mathbb{H}$ are proved in a similar way as the case without symmetry.

\section{(d) Special cases: $G=1$ and $G=\mathrm{SO}(n)$}

Example: $G=1$ Suppose that $f: X \rightarrow X$ is a topologically mixing Axiom A diffeomorphism. Then there is an open and dense set of mean zero functions $\Phi$ : $X \rightarrow \mathbb{R}^{n}$ in the $C^{r}$ topology (for each $r \geq 1$ ) such that $\frac{1}{\sqrt{N}} \sum_{j=0}^{N-1} \Phi \circ f^{j}$ converges in distribution to a general nondegenerate $n$-dimensional normal distribution with mean zero. That is, for each cube $I \subset \mathbb{R}^{n}$,

$$
\lim _{N \rightarrow \infty} m\left\{y \in X: \frac{1}{\sqrt{N}} \sum_{j=0}^{N-1} \Phi\left(S^{j}(y)\right) \in I\right\}=\frac{1}{(2 \pi)^{n / 2}(\operatorname{det} \Sigma)^{1 / 2}} \int_{I} \exp \left\{-\frac{1}{2}\left\langle\Sigma^{-1} x, x\right\rangle\right\} d x,
$$

where $\Sigma$ is a general $n \times n$ covariance matrix. 
Example: $G=\mathbf{S O}(n)$ Suppose that $f: X \rightarrow X$ is a topologically mixing Axiom A diffeomorphism, and that $G=\mathbf{S O}(n)$ acts in the standard way on $\mathbb{R}^{n}$. If $n=2$, suppose further that $f$ is Anosov. Then there is an open and dense set of $\mathbf{S O}(n)$ extensions $S: X \times \mathbf{S O}(n) \rightarrow X \times \mathbf{S O}(n)$ and $\mathbf{S O}(n)$-equivariant functions $\Phi:$ $X \times \mathbf{S O}(n) \rightarrow \mathbb{R}^{n}$ in the $C^{r}$ topology (for each $r \geq 1$ ) such that $\frac{1}{\sqrt{N}} \sum_{j=0}^{N-1} \Phi \circ S^{j}$ converges in distribution to a nondegenerate $n$-dimensional normal distribution with mean zero and covariance matrix $\sigma^{2} I_{n}$ where $\sigma>0$. That is, for each cube $I \subset \mathbb{R}^{n}$,

$$
\lim _{N \rightarrow \infty} m\left\{y \in X \times \mathbf{S O}(n): \frac{1}{\sqrt{N}} \sum_{j=0}^{N-1} \Phi\left(S^{j}(y)\right) \in I\right\}=\frac{1}{\left(2 \pi \sigma^{2}\right)^{n / 2}} \int_{I} e^{-|x|^{2} / 2 \sigma^{2}} d x .
$$

\section{Extensions of hyperbolic base dynamics}

In Section 4(b), we gave conditions under which $\epsilon(N)$ is unbounded. We now specialize to the case when the underlying dynamics is Axiom A. Using the equivariant central limit theorems established in Section 5, we investigate convergence in distribution of $\frac{1}{\sqrt{N}} \epsilon(N)$ to a nondegenerate $n$-dimensional normal distribution.

Throughout this section, $X$ is a compact Riemannian manifold and the base dynamics $f: X \rightarrow X$ is a topologically mixing Axiom A diffeomorphism when restricted to a hyperbolic basic set equipped with a Gibbs measure $\mu$. Let $\Gamma=G \ltimes \mathbb{R}^{n}$ where $G$ is a compact Lie group and define the product measure $m=\mu \times \nu_{G}$ on $X \times G$. We consider $\Gamma$-extensions $T: X \times \Gamma \rightarrow X \times \Gamma$. As in Section 5, our main results require that hypothesis $(\mathrm{H})$ is valid.

We view the $\Gamma$-extension $T$ as an $\mathbb{R}^{n}$-extension of the $G$-extension $S: X \times G \rightarrow$ $X \times G$. Let $\left\{v_{x, g}(N), N \geq 1\right\}$ denote the $\mathbb{R}^{n}$ component of the trajectory on $X \times \Gamma$ with initial condition $(x, g, 0)$. By Proposition 2.1,

$$
v_{x, g}(N)=\rho_{g} \sum_{j=0}^{N-1} \rho_{h_{j}(x)} k\left(f^{j}(x)\right)
$$

where $h_{j}(x)=h(x) h(f(x)) \cdots h\left(f^{j-1}(x)\right)$. Recall that we write

$$
v_{x, g}(N)=N \bar{v}+\epsilon_{x, g}(N),
$$

where $\bar{v}$ is the mean growth and $\epsilon(N)$ is sublinear.

Theorem 6.1 Let $f: X \rightarrow X$ be Axiom $A$, and $\Gamma=G \ltimes \mathbb{R}^{n}$ where $G$ is a compact connected Lie group. Suppose that hypothesis (H) is satisfied.

Then there is an open and dense set of $\Gamma$-extensions $T: X \times \Gamma \rightarrow X \times \Gamma$ in the

$C^{r}$ topology (for each $r \geq 1$ ) such that $\frac{1}{\sqrt{N}} \epsilon(N)$ converges in distribution to a general nondegenerate $G$-equivariant $n$-dimensional normal distribution with mean zero. 
Proof Consider the $G$-equivariant function $\Phi: X \times G \rightarrow \mathbb{R}^{n}$ given by $\Phi(x, g)=$ $\rho_{g} k(x)-\bar{v}$. By definition, $\bar{v}=\int_{X \times G} \rho_{g} k(x) d m$ so that $\Phi$ has mean zero. Moreover, $\epsilon_{y}(N)=\sum_{j=0}^{N-1} \Phi\left(S^{j}(y)\right)$. Now apply Theorem 5.11.

Remark 6.2 In the special cases $\Gamma=\mathbb{R}^{n}$ and $\Gamma=\mathbf{S E}(n)$, we obtain the more precise results described in subsection $5(\mathrm{~d})$.

\section{Extensions of quasiperiodic base dynamics}

In this section we consider smooth noncompact group extensions of quasiperiodic flows and maps. As usual, we restrict attention to groups of the form $\Gamma=G \ltimes \mathbb{R}^{n}$ where $G$ is a compact connected Lie group.

The base dynamics takes the form of an irrational torus flow $\dot{\theta}=\alpha$ on $T^{m}$ or an irrational torus map $f(\theta)=R_{\alpha} \theta=\theta+\alpha$. The results depend heavily on whether $\alpha$ is Diophantine or non-Diophantine. For example, suppose that $\Gamma=\mathbf{S E}(2)$ in the context of flows. Then for almost all $\alpha$ (Diophantine) the dynamics on $T^{m} \times \mathbf{S E}(2)$ is bounded for almost every sufficiently smooth extension. In contrast, there is a generic set of $\alpha$ (non-Diophantine) for which the dynamics on $T^{m} \times \mathbf{S E}(2)$ is generically unbounded in the $C^{r}$ topology for any $r \geq 1$.

Subsection (a) contains some basic estimates involving Fourier coefficients and Diophantine approximations. In subsection (b), we consider the case when $\alpha$ is Diophantine. In subsection (c), we consider the case when $\alpha$ is non-Diophantine. We note that our results in subsection (b) are restricted to the case of flows with $G$ abelian.

\section{(a) Preliminaries}

The results in this section rely heavily on certain estimates on the magnitude of Fourier coefficients and on certain Diophantine inequalities.

Proposition 7.1 Suppose that $\phi: T^{m} \rightarrow \mathbb{R}$ has Fourier series $\phi(\theta)=\sum_{j \in \mathbb{Z}^{m}} a_{j} e^{2 \pi i\langle j, \theta\rangle}$ where $a_{-j}=\bar{a}_{j}$. Let $r \geq 1$.

(a) If $\phi$ is $C^{r}$, then $a_{j}=O\left(|j|^{-r}\right)$.

(b) If $a_{j}=O\left(|j|^{-d}\right)$ where $d>r+m$, then $\phi$ is $C^{r}$. Moreover, the Fourier series for partial derivatives up to and including order $r$ are uniformly convergent.

Proof These estimates are standard when $m=1$, see for example [9]. They are also well-known when $m>1$, but are recorded here for convenience. Part (a) follows from integration by parts just as in the case $m=1$. Indeed, $a_{j}=o\left(|j|^{-r}\right)$. 
To verify part (b), write the Fourier series in the form $\sum_{J \geq 0} \sum_{|j|=J} a_{j} e^{i\langle j, \theta\rangle}$. Consider the partial derivative $\frac{\partial^{r}}{\partial \theta_{1}^{r_{1}} \cdots \partial \theta_{m}^{r_{m}}}$ where $r_{1}+\cdots+r_{m}=r$. We apply this differential operator term by term to the Fourier series and show that the resulting series converges uniformly. The $J$ 'th term becomes $b_{J}=\sum_{|j|=J} a_{j}^{\prime} e^{i\langle j, \theta\rangle}$, where $a_{j}^{\prime}=i^{r} j_{1}^{r_{1}} \cdots j_{m}^{r_{m}} a_{j}$. Hence

$$
\left|b_{J}\right| \leq n_{J} \max _{|j|=J}\left|a_{j}^{\prime}\right| \leq n_{J} J^{r} \max _{|j|=J}\left|a_{j}\right|
$$

where $n_{J}$ is the number of vector integers $j \in \mathbb{Z}^{m}$ satisfying $|j|=J$. The estimates $\left|a_{j}\right|=O\left(|j|^{-d}\right)$ and $n_{J}=O\left(J^{m-1}\right)$ guarantee that $\left|b_{J}\right| \leq O\left(J^{-(d-r-m+1)}\right)$. Since $d>r+m$, the series $\sum_{J \geq 0} b_{J}$ is uniformly convergent as required.

Proposition 7.2 (a) Let $\nu>0$. Then for almost every $\alpha \in \mathbb{R}^{m}$, there exists a constant $c>0$ such that

$$
|\langle j, \alpha\rangle| \geq c|j|^{-(m+\nu)}, \quad \text { for all nonzero } j \in \mathbb{Z}^{m} \text {. }
$$

(b) Let $\alpha \in \mathbb{R}^{m}$ and $\nu>0$. Then for almost every $b_{0} \in \mathbb{R}$, there exists a constant $c>0$ such that

$$
\left|b_{0}+\langle j, \alpha\rangle\right| \geq c|j|^{-(m+\nu)}, \quad \text { for all nonzero } j \in \mathbb{Z}^{m} \text {. }
$$

Equations (7.1) and (7.2) are referred to as Diophantine conditions and $\alpha$ satisfying Proposition 7.2(a) and the pair $\alpha, b_{0}$ satisfying Proposition 7.2(b) are termed Diophantine. If $\alpha$ is not Diophantine we have the following result.

Proposition 7.3 Let $\nu>0$. Let $D \subset \mathbb{R}^{m}$ be the set of vectors $\alpha \in \mathbb{R}^{m}$ such that

$$
|\alpha-p / q|<q^{-\nu}
$$

has infinitely many solutions $p \in \mathbb{Z}^{m}, q \geq 1$. Then $D$ is a dense $G_{\delta}$ in $\mathbb{R}^{m}$.

Proof For each $q \geq 1$, define $B_{q}=\left\{\alpha \in \mathbb{R}^{m}:|\alpha-p / q|<q^{-\nu}\right.$, for some $\left.p \in \mathbb{Z}^{m}\right\}$. Define $C_{n}=\cup_{q \geq n} B_{q}$, for $n \geq 1$. It is immediate that $C_{n}$ is open in $\mathbb{R}^{m}$, and $C_{n}$ is also dense since $\mathbb{Q}^{m} \subset C_{n}$ for all $n$. Thus $D=\cap_{n \geq 1} C_{n}$ is a dense $G_{\delta}$. 


\section{(b) Diophantine quasiperiodic base}

In this subsection, we show that for sufficiently smooth $\mathbf{S E}(2)$-extensions of quasiperiodic flows, it is almost always the case (in the measure theoretic sense) that trajectories are bounded.

Theorem 7.4 Consider the irrational torus flow $\dot{\theta}=\alpha$ on $T^{m}$. Then, for almost every $\alpha \in \mathbb{R}^{m}$, and for almost every sufficiently smooth $\operatorname{SE}(2)$-extension $(h, k)$ : $T^{m} \rightarrow \mathbf{S E}(2)=\mathbf{S O}(2) \ltimes \mathbb{R}^{2}$, the dynamics on $T^{m} \times \mathbf{S E}(2)$ is bounded.

More precisely, let $b_{0}$ denote the 0 'th Fourier coefficient of $h$. Then it is sufficient that $h$ is $C^{4 m+2}$, that $k$ is $C^{2 m+1}$, and that $\alpha$ and $b_{0}$ satisfy the Diophantine conditions (7.1) and (7.2) respectively, with $\nu \in(0,1)$.

Proof We can write the SE(2)-extension in the form

$$
\dot{\theta}=\alpha, \quad \dot{\phi}=h(\theta(t)), \quad \dot{v}=e^{i \phi(t)} k(\theta(t)) .
$$

In particular, the $\dot{\phi}$ equation is given by

$$
\dot{\phi}=\sum_{j \in \mathbb{Z}^{m}} b_{j} e^{i\langle j, \theta(t)\rangle}=\sum_{j \in \mathbb{Z}^{m}} b_{j} e^{i\langle j, \alpha\rangle t},
$$

where $b_{j} \in \mathbb{C}, b_{-j}=\overline{b_{j}}$. Since $h$ is smooth, we can integrate term by term to obtain

$$
\phi(t)=b_{0} t+R(\theta(t))
$$

where $R: T^{m} \rightarrow \mathbb{R}$ is given by $R(\theta)=\sum_{j \neq 0} \frac{b_{j}}{i\langle j, \alpha\rangle} e^{i\langle j, \theta\rangle}$.

Now, we define a function $S: T^{m} \rightarrow \mathbb{C}$ given by $S(\theta)=e^{i R(\theta)} k(\theta)$. As shown below, our hypotheses guarantee that $S$ is smooth and hence can be written as a Fourier series $S(\theta)=\sum_{j \in \mathbb{Z}^{m}} d_{j} e^{i\langle j, \theta\rangle}$, with coefficients $d_{j} \in \mathbb{C}$. The $\dot{v}$ equation then takes the form

$$
\dot{v}=e^{i \phi(t)} k(\theta(t))=e^{i b_{0} t} e^{i R(\theta(t))} k(\theta(t))=e^{i b_{0} t} \sum_{j \in \mathbb{Z}^{m}} d_{j} e^{i\langle j, \alpha\rangle t} .
$$

Integration yields

$$
v(t)=\sum_{j \in \mathbb{Z}^{m}} \frac{d_{j}}{i\left(b_{0}+\langle j, \alpha\rangle\right)} e^{i\langle j, \alpha\rangle t} .
$$

We claim that $S$ is smooth (justifying the formal calculations above) and moreover that $v(t)$ is bounded as required. 
It remains to verify the claim. By Proposition 7.1(a), $\left|b_{j}\right|=O\left(|j|^{-(4 m+2)}\right)$. Since $\alpha$ satisfies equation (7.1) for some $c>0$, we have that $\left|\frac{b_{j}}{\langle j, \alpha\rangle}\right|=O\left(|j|^{-(3 m+2-\nu)}\right)$ for all $j$. By Proposition 7.1(b), $R$ is $C^{2 m+1}$. Since $k$ is $C^{2 m+1}$, we see that $S$ is $C^{2 m+1}$ verifying the first part of the claim. Moreover, $\left|d_{j}\right|=O\left(|j|^{-(2 m+1)}\right)$. This estimate combined with condition (7.2) yields

$$
\left|\frac{d_{j}}{b_{0}+\langle j, \alpha\rangle}\right|=O\left(|j|^{-(m+1-\nu)}\right) .
$$

It follows as in the proof of Proposition 7.1(b) that the trigonometric series (7.4) for $v(t)$ is uniformly convergent and hence that $v(t)$ is bounded.

Remark 7.5 The set of $\alpha$ and $b_{0}$ for which the theorem is valid can be made larger by increasing $\nu$ to be any positive constant, at the cost that we require more regularity for the extension $(h, k)$.

Remark 7.6 Theorem 7.4 has applications to certain bifurcations of spiral waves considered in [15]. The symmetry group is $\mathbf{S E}(2)$ and there is a transition from periodic base dynamics (meandering spirals) to quasiperiodic base dynamics. (Due to drifts along the $\mathbf{S E}(2)$ group orbits, this is a transition generically from a two frequency motion to a three frequency motion.) Sandstede et al. [15] point out that the quasiperiodic base dynamics leads to typically sublinear growth, whereas we predict typically bounded growth.

The methods in this subsection extend directly to groups $\Gamma=G \ltimes \mathbb{R}^{n}$ for which $G$ is compact and abelian. As in the case of extensions of periodic flows, the results depend on whether $\operatorname{Fix}(G)=\{0\}$ (for example, $\Gamma=\operatorname{SE}(2)$ ) or $\operatorname{Fix}(G) \neq\{0\}$ (for example $\Gamma=\mathbb{R}^{n}$ ).

Theorem 7.7 Consider the irrational torus flow $\dot{\theta}=\alpha$ on $T^{m}$, where $\alpha$ is Diophantine. Let $\Gamma=G \ltimes \mathbb{R}^{n}$ where $G$ is a compact abelian Lie group and suppose that $\zeta: T^{m} \rightarrow \Gamma$ is sufficiently smooth $\left(C^{2 m+4}\right.$ is sufficient). Then

(a) The $\mathbb{R}^{n}$ component of each trajectory can be written as $v(t)=\bar{v} t+\epsilon(t)$ where $\bar{v} \in \mathbb{R}^{n}$ and $\epsilon(t)$ is bounded,

(b) If $\operatorname{Fix}(G)=\{0\}$ then $\bar{v}=0$ for almost every extension $\zeta$, and

(c) If $\operatorname{Fix}(G) \neq\{0\}$ then $\bar{v} \neq 0$ for almost every extension $\zeta$. 
Proof Write $G=\mathbf{T}^{s}$ where $s \geq 1$ and write $g=\left(\phi_{1}, \ldots, \phi_{s}\right) \in G$. The irreducible representations of $G$ have dimension either one or two. We consider one by one the irreducible representations for the action of $G$ on $\mathbb{R}^{n}$. It is sufficient to prove that

(i) if $G$ acts irreducibly but nontrivially on $\mathbb{R}^{2}$ then typically $v(t)$ is bounded; and (ii) if $G$ acts trivially on $\mathbb{R}$ then typically $v(t)=\bar{v}(t)+\epsilon(t)$ where $\bar{v} \neq 0$ and $\epsilon(t)$ is bounded.

In case (i), the proof proceeds in identical manner to the proof of Theorem 7.4. The $\dot{v}$ equation takes the form

$$
\dot{v}=e^{i\left(p_{1} \phi_{1}(t)+\cdots+p_{s} \phi_{s}(t)\right)} k(\theta(t)),
$$

where $p_{1}, \ldots, p_{s} \in \mathbb{Z}$ are not all zero. Let $b_{0, i}$ denote the constant term in the Fourier series for $\dot{\phi}_{i}$. Then,

$$
\dot{v}=e^{i\left(p_{1} b_{0,1}+\cdots+p_{s} b_{0, s}\right) t} \sum_{j \in \mathbb{Z}^{m}} d_{j} e^{i\langle j, \alpha\rangle t},
$$

where $d_{j} \in \mathbb{C}$. Hence for almost every choice of $b_{0,1}, \ldots, b_{0, s}$, there are no constant terms in the expansion of $\dot{v}$. As in the proof of Theorem 7.4,v(t) is almost always bounded.

In case (ii), $\dot{v}=\sum_{j \in \mathbb{Z}^{m}} d_{j} e^{i\langle j, \alpha\rangle t}$. Hence $\bar{v}=d_{0}$ which is typically nonzero, and $v(t)-d_{0} t$ is almost always bounded as before.

Remark 7.8 (a) Comparing Theorem 7.7 with Proposition 3.1, we see that the conclusions for sufficiently smooth extensions of Diophantine quasiperiodic flows with $G$ abelian are identical to the conclusions for extensions of periodic flows.

(b) The results in this subsection do not easily extend to maps. However, the case of degree zero extensions of quasiperiodic maps follows immediately from the results for flows.

\section{(c) Non-Diophantine quasiperiodic base}

We have seen that typically (with probability one) trajectories are bounded for smooth $\mathbf{S E}(2)$ extensions of Diophantine quasiperiodic flows. In contrast, we show that generically trajectories are unbounded (cf. Definition 4.1) for smooth extensions of nonDiophantine quasiperiodic flows (and maps). It is convenient to prove the result first for maps. The result for flows follows immediately.

The idea behind the proof is (i) that non-Diophantine quasiperiodic dynamics can be approximated by periodic dynamics of high period, and (ii) for certain compact Lie groups $G$, we can perturb $G$-extensions of periodic dynamics of high enough period $q$ so that the dynamics for the $G$-extension is periodic of period $q$ (cf. Section 4(a)). 
Proposition 7.9 Suppose that $G$ is a compact manifold. Let $r \geq 1$. Then there is a constant $D>0$ with the following property. Suppose that there are finite sets of points $\left\{x_{i}\right\} \in T^{m}$ distinct, and $\left\{g_{i}\right\} \in G$. Let $d=\min _{i \neq j}\left|x_{i}-x_{j}\right|>0$. Then there is a smooth function $\eta: T^{m} \rightarrow G$ such that $\eta\left(x_{i}\right)=g_{i}$ for all $i$ and $|\eta|_{r} \leq D / d^{r}$.

Proof First, consider the similar problem with $T^{m}$ replaced by $\mathbb{R}^{m}$ and $q=1$. Since $G$ is compact, it is clear that there is a uniform constant $C_{r}>0$, depending only on $G$ and $r$, such that there is a smooth function $f: \mathbb{R}^{m} \rightarrow G$ with specified values at sets of points separated by distance at least one, such that $|f|_{r} \leq C_{r}$. Now consider the function $\eta(y)=f(y / d)$. Then $\eta$ takes specified values at sets of points separated by distance at least $d$ and $|\eta|_{r} \leq D / d^{r}$ where $D=\max \left\{C_{1}, \ldots, C_{r}\right\}$. This result transfers to $T^{m}$ with the obvious modifications.

Lemma 7.10 Let $f=R_{p / q}: T^{m} \rightarrow T^{m}$ be a rational torus map where $p \in \mathbb{Z}^{m}$, $q \geq 1$. Let $\Gamma=G \ltimes \mathbb{R}^{n}$ where $G$ is abelian. Suppose that $\zeta=(h, k): T^{m} \rightarrow \Gamma$ is a $C^{r}$ extension, where $r \geq 1$.

Fix $x_{0} \in T^{m}$, and let $\delta>0$. Suppose that $q>\operatorname{diam} G / \delta$. Then there exists a $C^{r}$ extension $\widetilde{\zeta}=(\widetilde{h}, \widetilde{k}): T^{m} \rightarrow \Gamma$ such that $\left|\widetilde{\zeta} \zeta^{-1}\right|_{r}<\delta$ and

$$
\left|\widetilde{k}_{\ell q}\left(x_{0}\right)-k_{\ell q}\left(x_{0}\right)\right|=\frac{\delta \ell}{D q^{r-1}},
$$

for all $\ell \geq 1$, where $D$ is a constant that depends only on $r$ and $\Gamma$.

Proof Since $x_{0}$ is $q$-periodic and $G$ is abelian, it follows, as in the proof of Theorem 4.4 , that there is a small constant perturbation $\widetilde{h}$ of $h$ such that $\widetilde{h}_{q}\left(x_{0}\right)=e$. It is clear that the perturbation can be chosen so that $|\widetilde{h}-h|_{r}=|\widetilde{h}-h|_{0}<\operatorname{diam} G / q<\delta$.

Let $\eta\left(f^{j} x_{0}\right)=\rho_{h_{j}\left(x_{0}\right)}^{-1}, 0 \leq j \leq q$. The points $f^{j}\left(x_{0}\right)$ are equally spaced at distances $1 / q$ from each other, and the image of $\rho: G \rightarrow L\left(\mathbb{R}^{n}\right)$ is compact, so it follows from Proposition 7.9 that $\eta$ extends to a smooth function $\eta: T^{m} \rightarrow L\left(\mathbb{R}^{n}\right)$ such that $|\eta|_{r} \leq D q^{r}$ for some constant $D$ that depends only on $r$ and $\Gamma$.

Now, let $w \in \mathbb{R}^{n}$ be a vector of unit length and define $\widetilde{k}(x)=k(x)+\frac{\delta}{D q^{r}} \eta(x) w$. Then $|\widetilde{k}-k|_{r}<\delta$. By Proposition 2.1,

$$
\begin{aligned}
\widetilde{k}_{q}\left(x_{0}\right) & =k_{q}\left(x_{0}\right)+\frac{\delta}{D q^{r}}\left\{\eta\left(x_{0}\right) w+\rho_{\widetilde{h}_{1}\left(x_{0}\right)} \eta\left(f\left(x_{0}\right)\right) w+\cdots+\rho_{\widetilde{h}_{q-1}\left(x_{0}\right)} \eta\left(f^{q-1}\left(x_{0}\right)\right) w\right\} \\
& =k_{q}\left(x_{0}\right)+\frac{\delta}{D q^{r}}\{w+\cdots+w\}=k_{q}\left(x_{0}\right)+\frac{\delta}{D q^{r-1}} w .
\end{aligned}
$$

Since $\widetilde{h}_{q}\left(x_{0}\right)=e$, it follows, as in the proof of Theorem 4.4 , that $\widetilde{k}_{q \ell}\left(x_{0}\right)=k_{q \ell}\left(x_{0}\right)+$ $\frac{\delta \ell}{D q^{r-1}} w$ for all $\ell \geq 1$ as required. 
Theorem 7.11 Let $f=R_{\alpha}: T^{m} \rightarrow T^{m}$ be an irrational torus map. Let $\Gamma=G \ltimes \mathbb{R}^{n}$ where $G$ is abelian, and fix $r \geq 1$. Then for a residual set of $\alpha \in \mathbb{R}^{m}$ there exists a residual set of $C^{r}$ extensions $\zeta: T^{m} \rightarrow \Gamma$ for which the dynamics on $T^{m} \times \Gamma$ is unbounded.

More precisely, choose $\nu>3(r+1)$. If $\alpha$ is such that the inequality (7.3) has infinitely many solutions then the dynamics is unbounded for a residual set of extensions $\zeta$ in the $C^{r}$ topology.

Proof Let $\zeta=(h, k): T^{m} \rightarrow \Gamma$. We have the $\Gamma$-extension $T_{\alpha}(x, g, v)=\left(R_{\alpha} x, g h(x), v+\right.$ $\left.\rho_{g} k(x)\right)$. Also, for any $p \in \mathbb{Z}^{m}, q \geq 1$, we have the $\Gamma$-extension $T_{p / q}(x, g, v)=$ $\left(R_{p / q} x, g h(x), v+\rho_{g} k(x)\right)$. Taking iterates, we have

$$
T_{\alpha}^{j}(x, e, 0)=\left(R_{j \alpha} x, h_{j, \alpha}(x), k_{j, \alpha}(x)\right),
$$

and similarly for $T_{p / q}^{j}$. Here, $h_{j, *}$ and $k_{j, *}$ are defined in the usual way (cf. Proposition 2.1) but we require the additional subscript to indicate the base transformation.

We claim that there exists a constant $C$, independent of $\alpha, p, q$ and $j$, such that

$$
\left|k_{j, \alpha}-k_{j, p / q}\right| \leq C j^{3}|\alpha-p / q| \text {. }
$$

We show how the result follows from the claim. Let $\delta>0$ and $M>0$, and choose $x_{0} \in T^{m}$. We show that there is a perturbation of $\zeta$ that is $\delta$-small in the $C^{r}$ topology such that the perturbed extension $\zeta=(h, k)$ satisfies $\left|k_{j, \alpha}\left(x_{0}\right)\right|>M$ for some $j \geq 1$. It follows that the set of extensions with unbounded trajectories is a countable intersection of open and dense subsets.

Fix $r \geq 1$ and suppose that $\nu>3(r+1)$. Choose $q \geq 1$ such that

$$
q>\frac{\operatorname{diam} G}{\delta}, \quad \frac{\delta q}{2 D}>2 M \quad \text { and } \quad \frac{C}{q^{\nu-3(r+1)}}<M .
$$

(Here, $D>0$ is defined in Lemma 7.10.) Since inequality (7.3) has infinitely many integer solutions, we can choose $q \geq 1$ satisfying inequalities (7.6) and $p \in \mathbb{Z}^{m}$ such that $|\alpha-p / q|<1 / q^{\nu}$. Taking $j=q^{r+1}$ in the claim, we have that

$$
\left|k_{q^{r+1}, \alpha}-k_{q^{r+1}, p / q}\right| \leq C\left(q^{r+1}\right)^{3} / q^{\nu}=C / q^{\nu-3(r+1)}<M .
$$

By (7.6), we have $q>\operatorname{diam} G / \delta$ and so can apply Lemma 7.10. Taking $\ell=q^{r}$ in (7.5), we can make a perturbation of size less than $\delta$ in the $C^{r}$ topology so that $\left|k_{q^{r+1}, p / q}\left(x_{0}\right)\right| \geq \frac{\delta q}{2 D}>2 M$. Hence

$$
\left|k_{q^{r+1}, \alpha}\left(x_{0}\right)\right| \geq\left|k_{q^{r+1}, p / q}\left(x_{0}\right)\right|-\left|k_{q^{r+1}, \alpha}\left(x_{0}\right)-k_{q^{r+1}, p / q}\left(x_{0}\right)\right|>2 M-M=M
$$


as required.

It remains to verify the claim. Let $|h|_{d}$ and $|k|_{d}$ denote the norms of $h$ and $k$ in the $C^{d}$ topology. By Proposition 2.1,

$$
\begin{aligned}
\left|k_{j, \alpha}(x)-k_{j, p / q}(x)\right|= & \left|\sum_{s=0}^{j-1}\left(\rho_{h_{s, \alpha}(x)} k(x+s \alpha)-\rho_{h_{s, p / q}(x)} k(x+s p / q)\right)\right| \\
\leq & \sum_{s=0}^{j-1}\left|\rho_{h_{s, \alpha}(x)} k(x+s \alpha)-\rho_{h_{s, p / q}(x)} k(x+s \alpha)\right| \\
& \quad+\sum_{s=0}^{j-1}\left|\rho_{h_{s, p / q}(x)} k(x+s \alpha)-\rho_{h_{s, p / q}(x)} k(x+s p / q)\right| \\
\leq & \sum_{s=0}^{j-1}\left|\rho_{h_{s, \alpha}(x)}-\rho_{h_{s, p / q}(x)}\right||k|_{0}+\sum_{s=0}^{j-1}|k|_{1} s|\alpha-p / q| .
\end{aligned}
$$

Now $h_{s, \alpha}(x)=h_{s-1, \alpha}(x) h(x+(s-1) \alpha)$ and similarly for $h_{s, p / q}$. Hence

$$
\begin{aligned}
\left|\rho_{h_{s, \alpha}(x)}-\rho_{h_{s, p / q}(x)}\right|= & \left|\rho_{h_{s-1, \alpha}(x)} \rho_{h(x+(s-1) \alpha)}-\rho_{h_{s-1, p / q}(x)} \rho_{h(x+(s-1) p / q)}\right| \\
\leq & \left|\rho_{h_{s-1, \alpha}(x)} \rho_{h(x+(s-1) \alpha)}-\rho_{h_{s-1, p / q}(x)} \rho_{h(x+(s-1) \alpha)}\right| \\
& \quad+\left|\rho_{h_{s-1, p / q}(x)} \rho_{h(x+(s-1) \alpha)}-\rho_{h_{s-1, p / q}(x)} \rho_{h(x+(s-1) p / q)}\right| \\
\leq & \left|\rho_{h_{s-1, \alpha}(x)}-\rho_{h_{s-1, p / q}(x)}\right|+|\rho||h|_{1}(s-1)|\alpha-p / q| .
\end{aligned}
$$

It follows by induction that

$$
\left|\rho_{h_{s, \alpha}(x)}-\rho_{h_{s, p / q}(x)}\right| \leq \frac{1}{2}(s-1)(s-2)|\rho|\left|h_{1}\right||\alpha-p / q| .
$$

Hence

$$
\begin{aligned}
\left|k_{j, \alpha}(x)-k_{j, p / q}(x)\right| & \leq \sum_{s=0}^{j-1} \frac{1}{2}(s-1)(s-2)|\rho||h|_{1}|\alpha-p / q|+\sum_{s=0}^{j-1} s|k|_{1}|\alpha-p / q| \\
& \leq\left(j^{3}|\rho||h|_{1}+j^{2}|k|_{1}\right)|\alpha-p / q| \leq C j^{3}|\alpha-p / q|,
\end{aligned}
$$

for some constant $C>0$. This proves the claim.

Remark 7.12 (a) As in Section 4, we can replace the condition that $G$ is abelian by the condition that $\operatorname{Fix}(\mathbf{T}) \neq\{0\}$ where $\mathbf{T}$ is a maximal torus in $G$. Thus, for the Euclidean groups $\Gamma=\mathbf{S E}(n)$, our result applies to the cases $n=2$ and $n$ odd. We conjecture (cf. Remark 4.6(b)) that Theorem 7.11 holds for $G$ an arbitrary compact Lie group.

(b) For extensions of a quasiperiodic flow we take the time $\tau$ map and perturb the vector field to obtain the same result.

(c) When $r=1$, we require only that $\nu>3$ in Theorem 7.11. 
Acknowledgments We thank Dima Dolgopyat for helpful discussions and for generously making his paper available to us before publication. We are also grateful to Mike Field and Andrew Török for helpful discussions. IM was supported in part by NSF Grant DMS-9704980 and by EPSRC Grant GR/M22499. MN was supported by a grant from the Nuffield Foundation and by EPSRC grant GR/14117.

\section{References}

[1] P. Ashwin and I. Melbourne. Noncompact drift for relative equilibria and relative periodic orbits. Nonlinearity 10 (1997) 595-616.

[2] V. N. Biktashev and A. Holden. Deterministic Brownian motion in the hypermeander of spiral waves. Physica D 116 (1998) 342-354.

[3] P. Billingsley. Probability and Measure. Wiley, New York, 1986.

[4] K. Burns and A. Wilkinson. Stable ergodicity of skew products. Ann. scient. Éc. Norm. Sup. 32 (1999) 859-889.

[5] D. Dolgopyat. On mixing properties of compact group extensions of hyperbolic systems. Preprint.

[6] M. J. Field and W. Parry. Stable ergodicity of skew extensions by compact Lie groups. Topology 38 (1999) 167-187.

[7] M. Grayson, C. Pugh and M. Shub. Stably ergodic diffeomorphisms. Annals of Math. 140 (1994) 295-329.

[8] R. Jones and W. Parry. Compact abelian group extensions of dynamical systems II. Compositio Mathematica 25 (1972) 135-147.

[9] Y. Katznelson. An introduction to Harmonic Analysis, Dover, New York, 1976.

[10] C. Liverani. Central limit theorems for deterministic systems. (Eds. F. Ledrappier, J. Lewowics and S. Newhouse), Pitman Res. Notes 363 (1996) 56-75.

[11] A. N. Livšic. Cohomology of dynamical systems. Math. USSR Izvestija 6 (1972) 1278-1301.

[12] M. Noorani. Ergodicity and weak-mixing of homogeneous extensions of measurepreserving transformations with applications to Markov shifts. Monatsh. Math. 123 (1997), 149-170. 
[13] M. Pollicott and C. Walkden. Livšic theorems for connected Lie groups. Trans. Amer. Math. Soc. To appear.

[14] D. Ruelle. Thermodynamic Formalism. Encyclopedia of Math. and its Applications 5, Addison-Wesley, Mass., 1978.

[15] B. Sandstede, A. Scheel and C. Wulff. Bifurcation and dynamics of spiral waves. J. Nonlin. Sci. 9 (1999) 439-478.

[16] C. Wulff, J. S. W. Lamb and I. Melbourne. Bifurcation from relative periodic solutions. Ergod. Th. 86 Dynam. Sys. To appear.

[17] L-S. Young. Statistical properties of dynamical systems with some hyperbolicity. Annals of Math. 147 (1998) 585-650. 